\title{
Quasiparticle self-energy and many-body effective mass enhancement in a two-dimensional electron liquid
}

\author{
R. Asgari, ${ }^{1,2}$ B. Davoudi, ${ }^{1,2, *}$ M. Polini, ${ }^{1, \dagger}$ Gabriele F. Giuliani, ${ }^{3}$ M. P. Tosi, ${ }^{1}$ and G. Vignale ${ }^{4}$ \\ ${ }^{1}$ NEST-INFM and Classe di Scienze, Scuola Normale Superiore, I-56126 Pisa, Italy \\ ${ }^{2}$ Institute for Studies in Theoretical Physics and Mathematics, Tehran 19395-5531, Iran \\ ${ }^{3}$ Physics Department, Purdue University, West Lafayette, Indiana 47907, USA \\ ${ }^{4}$ Department of Physics and Astronomy, University of Missouri-Columbia, Columbia, Missouri 65211, USA
}

(Received 28 June 2004; revised manuscript received 22 October 2004; published 18 January 2005)

\begin{abstract}
Motivated by a number of recent experimental studies we have revisited the problem of the microscopic calculation of the quasiparticle self-energy and many-body effective mass enhancement in an unpolarized two-dimensional electron liquid. Our systematic study is based on the many-body local field theory and takes advantage of the results of the most recent diffusion Monte Carlo calculations of the static charge and spin response of the electron liquid. We report extensive calculations of both the real and imaginary parts of the quasiparticle self-energy. We also present results for the many-body effective mass enhancement and the renormalization constant over a broad range of electron densities. In this respect we critically examine the relative merits of the on-shell approximation, commonly used in weak coupling situations versus the actual self-consistent solution of the Dyson equation. We show that already for $r_{s} \simeq 3$ and higher, a solution of the Dyson equation proves necessary in order to obtain a well-behaved effective mass. Finally we find confirmation that the inclusion of both charge- and spin-density fluctuations beyond the random phase approximation is indeed crucial to get reasonable agreement with recent measurements.
\end{abstract}

DOI: 10.1103/PhysRevB.71.045323

PACS number(s): 71.10.Ca

\section{INTRODUCTION}

An interacting electron gas (EG) on a uniform neutralizing background is used as the reference system in most realistic calculations of electronic structure in condensed-matter physics. ${ }^{1-3}$ At zero temperature there are only two relevant parameters for a disorder-free, homogeneous EG in the absence of quantizing magnetic fields and spin-orbital coupling: (i) the usual Wigner-Seitz density parameter $r_{s}$ $=\left(\pi n_{2 \mathrm{D}} a_{B}^{2}\right)^{-1 / 2}, a_{B}=\hbar^{2} \bar{\kappa} /\left(m e^{2}\right)$, being the Bohr radius in the medium of interest with $\bar{\kappa}$ and $m$ appropriate dielectric constant and bare band mass, respectively; and (ii) the degree of spin polarization $\zeta=\left|n_{\uparrow}-n_{\downarrow}\right| / n_{2 \mathrm{D}}$. Here $n_{\sigma}$ is the average density of particles with spin $\sigma=\uparrow, \downarrow$, and $n_{2 \mathrm{D}}=n_{\uparrow}+n_{\downarrow}$ is the total average density.

Understanding the many-body aspects of this model has attracted continued interest for many decades. ${ }^{2-6}$ The EG, unlike systems of classical particles, behaves like an ideal paramagnetic gas at high density $\left(r_{s} \ll 1\right)$ and like a solid at low density ${ }^{7}\left(r_{s} \gg 1\right)$. In the intermediate density regime, which is relevant in three dimensions to conduction electrons in simple metals and in two dimensions, to electrons in an inversion layer of a $\mathrm{Si}$ metal-oxide-semiconductor fieldeffect transistor (MOSFET) or in an $\mathrm{AlGaAs} / \mathrm{GaAs}$ quantum well, perturbative techniques are not effective owing to the lack of a small expansion parameter. Therefore, one has to take recourse to approximate semianalytical methods, a number of which have been reviewed in Refs. 3 and 4 , or to quantum Monte Carlo (QMC) simulation methods. ${ }^{8-18}$

Among the methods designed to deal with the intermediate density regime, of particular interest for its physical appeal and elegance is Landau's phenomenological theory ${ }^{19}$ dealing with low-lying excitations in a Fermi liquid. Landau called such single-particle excitations quasiparticles (QPs) and postulated a one-to-one correspondence between them and the excited states of a noninteracting Fermi gas. He wrote the excitation energy of the Fermi-liquid in terms of the energies of the QPs and of their effective interaction. The QP-QP interaction function can, in turn, be used to obtain various physical properties of the system and can be parametrized in terms of experimentally measurable data.

Quinn and Ferrell ${ }^{20}$ provided a framework for the microscopic evaluation of the QP-QP interactions in the EG by means of the random phase approximation (RPA). Next, Rice $^{21}$ incorporated the vertex corrections in the RPA form of the electron self-energy by including the Hubbard ${ }^{22}$ manybody local field, while Overhauser ${ }^{5}$ discussed, albeit within a simplified framework, numerous quasiparticle properties using a similar approximation. Some of the problems in Rice's theory were subsequently resolved by Ting, Lee, and Quinn ${ }^{23}$ in their theory of the quasi-two-diemensional EG. All these approaches considered only one many-body local field, a procedure that does not allow for a correct handling of the effect of spin-density fluctuations. Ultimately this approximation leads to a number of serious problems that only the physically sound inclusion of the latter can resolve. ${ }^{24}$

A more detailed analysis, which accounts for the vertex corrections associated with both types of fluctuations, was carried out for an unpolarized EG in Refs. 25-28, where Kukkonen-Overhauser-like ${ }^{29}$ effective interactions were obtained by different approaches. In particular, Yarlagadda and Giuliani $^{27,28}$ adopted a physically transparent approach termed the renormalized Hamiltonian approach (RHA), which consisted of a generalization of the RPA-based elegant pioneering theory of Hamann and Overhauser. ${ }^{30}$ A few electrons from the EG are selected and called "test electrons," while the remaining EG is treated as a dielectric screening medium. As the test electrons move through this medium, 
they produce fluctuations in the density of spin-up and spindown electrons, which, in turn, provide virtual clothing and also screen their mutual interaction. Thus, the dielectric mimics the true physical processes in an average way. Of course, the test electrons and the electrons of the medium are physically indistinguishable, and this must be taken into account when exchange effects are considered. At this point, after averaging over the coordinates of the screening medium, an effective renormalized Hamiltonian containing only the degrees of freedom of the clothed test electrons (or QPs) can be derived, under the assumption that the coupling with the medium occurs only via its charge- and spin-density fluctuations. Calculations based on these theories have been carried out for both three-dimensional (3D) 26,31 and twodimensional (2D) 24,32,33 systems.

In a parallel theoretical development $\mathrm{Ng}$ and Singwi, ${ }^{34}$ starting from a Ward identity and performing a local approximation on the irreducible particle-hole interaction, obtained an expression for the self-energy in terms of the many-body local-field factors associated with charge- and spin-density fluctuations. Equivalent results were later obtained by Yarlagadda and Giuliani ${ }^{24,28}$ by means of the RHA. These authors also took into account an infinitesimal degree of spin polarization, which allowed them to properly carry out Rice's program of calculating the Landau Fermi-liquid parameters.

Although its theoretical basis is sound, previous quantitative theoretical work based on this general method suffers from two major shortcomings: (i) All available earlier calculations have adopted a static and oversimplified Hubbard-like model for the local-field factors, which do not have the appropriate behavior at both intermediate and large wave number $q$; and (ii) most theories (including the RHA introduced above and briefly reviewed in Sec. II C) are based on the "on-shell" approximation, which, as we will show in this work, predicts a spurious divergence of the effective mass with decreasing electron density. While still neglecting the frequency dependence of the local fields, we have corrected for several of these discrepancies. In particular we have implemented the following improvements: (i) we have made use of recent parametrizations ${ }^{35}$ for the static local fields of a 2D EG, and, in the spirit of the work of Santoro and Giuliani $^{32}$ and of $\mathrm{Ng}$ and Singwi, ${ }^{34}$ (ii) we have kept the full frequency dependence of the self-energy and carried out a self-consistent solution of the Dyson equation to find the proper QP excitation energy and QP properties. Moreover, comparing to Ref. 32, we have released the simplifying plasmon-plus-paramagnon-pole approximation to the chargecharge and spin-spin response functions. A calculation based on the same theory for the case of a $3 D$ EG is reported in Ref. 36.

From the experimental point of view, as already remarked, electrons in a semiconductor inversion layer or in a quantum well can be modeled by a quasi-2D EG. ${ }^{37}$ Quantum Shubnikov-de Haas ( $\mathrm{SdH}$ ) oscillations of the magnetoresistance $^{38}$ provide a powerful tool for measuring Fermiliquid parameters of a quasi-2D EG. Measurements performed over the past years ${ }^{39-42}$ have shown sizeable renormalizations of the QP effective-mass and effective-Landé $g$ factor. These experiments have been performed in a relatively high-density regime, i.e., for $r_{s} \lesssim 2$ say. The density dependence of the effective mass $m^{*}$ was obtained by Smith and Stiles $^{39}$ from a study of $\mathrm{SdH}$ oscillations in $\mathrm{Si}$ inversion layers. To obtain the same information Abstreiter et al. ${ }^{40}$ used instead cyclotron resonance measurements. Fang and Stiles $^{41}$ and Neugebauer et al. $^{42}$ performed a series of $\mathrm{SdH}$ experiments on $\mathrm{Si}$ inversion layers and obtained the dependence of the modified Landé factor $g^{*}$ on carrier density. The product of $g^{*}$ and $m^{*}$, which is proportional to the spin susceptibility $\chi_{S}$, can be determined from the SdH oscillations in a tilted magnetic field, as suggested in Ref. 41.

The issue of the apparent metal-insulator transition ${ }^{43}$ (MIT) in low-density 2D electron systems has prompted intense experimental studies on quasiparticle properties ${ }^{44-52,56}$ in the intermediate-to-strong coupling regime, $r_{s} \gtrsim 2$, for example. Many authors ${ }^{44}$ have shown that the resistance of a Si-MOSFET is increased dramatically by increasing the value of an in-plane magnetic field and saturates at a characteristic value of several Teslas. Performing low-field $\mathrm{SdH}$ measurements on Si-MOSFET's, Okamoto et al. ${ }^{45}$ have shown that the saturation value is the magnetic field necessary to fully polarize the electron spins. An interpretation ${ }^{46,47}$ of the in-plane magnetoresistance in Si inversion layers suggested a ferromagnetic instability at or very close to the critical density for the 2D MIT driven by a divergence in the effective mass. Direct measurements of $m^{*}$ in high-mobility Si-MOSFET's over a wide range of carrier density, using a technique based on the beating pattern of $\mathrm{SdH}$ oscillations in crossed magnetic fields, have been reported by Pudalov et $a l .{ }^{48}$ These authors measured $m^{*}$ and $\chi_{S}$ in the vicinity of the 2D MIT, but found no evidence for a divergent behavior. Only a moderate enhancement of $m^{*}$ by a factor of $\approx 2-2.5$ over the band mass was observed near the critical density for the 2D MIT. Two groups have also reported anomalous density dependences of the Landé factor in $n$-doped ${ }^{49}\left(2 \lesssim r_{s}\right.$ $\lesssim 7)$ and $p$-doped ${ }^{50}\left(r_{s} \gtrsim 17\right) \mathrm{GaAs} / \mathrm{AlGaAs}$ heterojunctions that are in disagreement with results in Si-MOSFETs. The dependence of the spin susceptibility on the degree of spin polarization of the sample can account for this anomalous behavior as pointed out by Zhu et al. ${ }^{51}$ who studied a 2D EG of exceedingly high quality.

To complete the cornucopia of recent experimental findings on QP properties, it is worth mentioning that Vakili et $a{ }^{52}$ have reported measurements of $m^{*}$ and $\chi_{S}$ in a dilute 2D EG confined to a narrow AlAs quantum well (only $45 \AA$ wide). The electron system investigated in Ref. 52 is quite interesting because the electrons occupy an out-of-plane conduction-band valley, rendering the system similar to $2 \mathrm{D}$ electrons in Si-MOSFETs but with only one valley occupied. Quite surprisingly, the results of Vakili et al. ${ }^{52}$ for $\chi_{S}$ are in good agreement with the QMC results of Attaccalite et al. ${ }^{18}$ even though this simulation has been carried out for a strictly disorder-free EG. This might indicate that $\chi_{S}$ is not strongly dependent on disorder. On the other hand, there is a significant spread in the experimental results of Ref. 52 for $m^{*}$, which turns out to be both sample and cool-down dependent. Difficulties associated with the $\mathrm{SdH}$ data analysis have been pointed out in Ref. 52 as one of the possible causes for this spread.

At this point it is probably worth commenting that, indeed, there could be, in principle, subtle issues associated 
with the analysis of the $\mathrm{SdH}$ traces in $2 \mathrm{D}$ systems. In fact the amplitude of the $\mathrm{SdH}$ oscillations is usually fitted to the Lifshitz-Kosevich (LK) formula ${ }^{53}$ upon a trivial change in the single-particle spectrum. The fit is based on an impurityscattering Dingle temperature $T_{D}$ and an "effective" mass. In recent years a number of caveats concerning the applicability of such a procedure to strongly interacting $2 \mathrm{D}$ systems have appeared. ${ }^{54}$ In particular, Martin et al. ${ }^{55}$ have shown that the interplay between electron-electron interactions and electronimpurity scattering leads in 2D to an effective temperaturedependent Dingle temperature with a leading lowtemperature behavior of the type $T_{D}(T) \propto T \ln T$. The need for the introduction of a temperature-dependent Dingle parameter in strongly coupled Si-MOSFETs has been emphasized in Ref. 48, where a linear $T_{D}(T)$ was used to fit the longitudinal magnetoresistance data. Quantitative differences on the resultant effective mass are found using such a procedure: roughly speaking, the tendency is to get substantially lower values for $m^{*}$ than those obtained using the same Dingle parameter for all temperatures.

For a quantitative comparison between suitable theories that take into account quasi-2D effects (such as finite width of the electron wave functions in the confinement direction and valley degeneracies) and the experimental results ${ }^{39,41}$ for Si-MOSFETs in the weak-coupling regime $r_{s} \leqslant 2$, we refer the reader to the work of Yarlagadda and Giuliani ${ }^{24}$ and references therein. In this work we will try and carry out a comparison between the theory and the experimental data of Tan et al. ${ }^{56}$ for strongly interacting electrons $\left(2 \lesssim r_{s} \lesssim 6\right)$, occupying a single valley in an exceptionally clean GaAs/AlGaAs quantum well.

The contents of the paper are described briefly as follows. In Sec. II we present in some detail, the theoretical background. We proceed in Sec. III to discuss the input we have used for our numerical calculations, while in Sec. IV we present our main results for the real and imaginary part of the quasiparticle self-energy, the many-body enhancement of the effective mass, and the renormalization constant. Finally, in Sec. V we compare our theory with the experimental results of Tan et al. ${ }^{56}$ and report some conclusions. In order to make the paper fully self-contained we have also included two appendixes, which contain a number of helpful details on how we have in practice calculated the QP self-energy.

\section{THEORY OF THE QUASIPARTICLE SELF-ENERGY}

The aim of this section is to provide the theoretical justification for the formulas we have made use of in our evaluation of the retarded QP self-energy $\Sigma_{\text {ret }}(\mathbf{k}, \omega)$ of a $2 \mathrm{D}$ paramagnetic EG. The main formulas are given in Eqs. (2) and (3). Their justification rests on both a diagrammatic perturbative analysis as well as on an effective quasiparticle Hamiltonian derived via a procedure based on the idea of renormalization.

\section{A. General formulas}

We will employ in our theory the following decomposition for the retarded QP self-energy $\Sigma_{\text {ret }}(\mathbf{k}, \omega)$ :

$$
\Sigma_{\text {ret }}(\mathbf{k}, \omega)=\Sigma_{\mathrm{SX}}(\mathbf{k}, \omega)+\Sigma_{\mathrm{CH}}(\mathbf{k}, \omega),
$$

where the first term is called "screened-exchange" (SX) and the second term is called "Coulomb-hole" $(\mathrm{CH})$. The frequency $\omega$ is measured from $\varepsilon_{F} / \hbar$.

The SX contribution is given by

$$
\Sigma_{\mathrm{SX}}(\mathbf{k}, \omega)=-\int \frac{d^{2} \mathbf{q}}{(2 \pi)^{2}} \frac{v_{\mathbf{q}}}{\varepsilon\left(\mathbf{q}, \omega-\xi_{\mathbf{k}+\mathbf{q}} / \hbar\right)} \Theta\left(-\xi_{\mathbf{k}+\mathbf{q}} / \hbar\right) .
$$

Here $\Theta(x)$ is the step function and $\xi_{\mathbf{k}}=\varepsilon_{\mathbf{k}}-\varepsilon_{F}$, where $\varepsilon_{\mathbf{k}}$ $=\hbar^{2} \mathbf{k}^{2} /(2 m)$ is the single-particle energy with $\varepsilon_{F}$ $=\hbar^{2} k_{F}^{2} /(2 m)$ and $k_{F}=\left(2 \pi n_{2 \mathrm{D}}\right)^{1 / 2}=\sqrt{2} /\left(r_{s} a_{B}\right)$, respectively, being the Fermi energy and wave number. The $\mathrm{CH}$ contribution to the retarded self-energy is, in turn, given by

$$
\Sigma_{\mathrm{CH}}(\mathbf{k}, \omega)=-\int \frac{d^{2} \mathbf{q}}{(2 \pi)^{2}} v_{\mathbf{q}} \int_{0}^{+\infty} \frac{d \Omega}{\pi} \frac{\operatorname{Im}\left[\varepsilon^{-1}(\mathbf{q}, \Omega)\right]}{\omega-\xi_{\mathbf{k}+\mathbf{q}} / \hbar-\Omega+i \delta},
$$

where $\delta$ is a positive infinitesimal. In Eqs. (2) and (3) $\varepsilon(\mathbf{q}, \omega)$ is a screening dielectric function originating from the effective Kukkonen-Overhauser interaction, ${ }^{28,29}$

$$
\begin{aligned}
\frac{1}{\varepsilon(\mathbf{q}, \omega)}= & 1+v_{\mathbf{q}}\left[1-G_{+}(\mathbf{q}, \omega)\right]^{2} \chi_{\mathrm{C}}(\mathbf{q}, \omega) \\
& +3 v_{\mathbf{q}} G_{-}^{2}(\mathbf{q}, \omega) \chi_{\mathrm{S}}(\mathbf{q}, \omega)
\end{aligned}
$$

where $v_{\mathbf{q}}=2 \pi e^{2} / q$ is the $2 \mathrm{D}$ Fourier transform of the bare Coulomb interaction $e^{2} / r$. In this expression $\chi_{\mathrm{C}}(\mathbf{q}, \omega)$ and $\chi_{\mathrm{S}}(\mathbf{q}, \omega)$ represent the charge-charge and spin-spin response functions, which, in turn, define and are determined by the spin-symmetric and spin-antisymmetric local-field factors $G_{+}(\mathbf{q}, \omega)$ and $G_{-}(\mathbf{q}, \omega)$ via the relations

$$
\chi_{\mathrm{C}}(\mathbf{q}, \omega)=\frac{\chi_{0}(\mathbf{q}, \omega)}{1-v_{\mathbf{q}}\left[1-G_{+}(\mathbf{q}, \omega)\right] \chi_{0}(\mathbf{q}, \omega)}
$$

and

$$
\chi_{\mathrm{S}}(\mathbf{q}, \omega)=\frac{\chi_{0}(\mathbf{q}, \omega)}{1+v_{\mathbf{q}} G_{-}(\mathbf{q}, \omega) \chi_{0}(\mathbf{q}, \omega)},
$$

where $\chi_{0}(\mathbf{q}, \omega)$ is the Lindhard response function of a noninteracting $2 \mathrm{D}$ EG. ${ }^{57}$ In the paramagnetic electron liquid $G_{ \pm}(\mathbf{q}, \omega)=\left[G_{\uparrow \uparrow}(\mathbf{q}, \omega) \pm G_{\uparrow \downarrow}(\mathbf{q}, \omega)\right] / 2$, where $G_{\sigma \sigma^{\prime}}(\mathbf{q}, \omega)$ are the spin-resolved local fields. Note that $\Sigma_{\mathrm{SX}}(\mathbf{k}, \omega)$ acquires the form of an ordinary exchange-like self-energy built from the Kukkonen-Overhauser effective interaction instead of the bare Coulomb interaction, which would lead to the familiar frequency-independent Hartree-Fock self-energy first calculated for the 2D EG by Chaplik. ${ }^{58}$

The real and imaginary part of the retarded self-energy are readily obtained from Eqs. (2) and (3) with the result 


$$
\begin{aligned}
\operatorname{Re} \Sigma_{\text {ret }}(\mathbf{k}, \omega)= & -\int \frac{d^{2} \mathbf{q}}{(2 \pi)^{2}} v_{\mathbf{q}} \operatorname{Re}\left[\varepsilon^{-1}\left(\mathbf{q}, \omega-\xi_{\mathbf{k}+\mathbf{q}} / \hbar\right)\right] \\
& \times \Theta\left(-\xi_{\mathbf{k}+\mathbf{q}} / \hbar\right)-\int \frac{d^{2} \mathbf{q}}{(2 \pi)^{2}} v_{\mathbf{q}} \mathrm{P} \\
& \times \int_{0}^{+\infty} \frac{d \Omega}{\pi} \frac{\operatorname{Im}\left[\varepsilon^{-1}(\mathbf{q}, \Omega)\right]}{\omega-\xi_{\mathbf{k}+\mathbf{q}} / \hbar-\Omega},
\end{aligned}
$$

and

$$
\begin{aligned}
\operatorname{Im} \Sigma_{\text {ret }}(\mathbf{k}, \omega)= & \int \frac{d^{2} \mathbf{q}}{(2 \pi)^{2}} v_{\mathbf{q}} \operatorname{Im}\left[\varepsilon^{-1}\left(\mathbf{q}, \omega-\xi_{\mathbf{k}+\mathbf{q}} / \hbar\right)\right] \\
& \times\left[\Theta\left(\omega-\xi_{\mathbf{k}+\mathbf{q}} / \hbar\right)-\Theta\left(-\xi_{\mathbf{k}+\mathbf{q}} / \hbar\right)\right] .
\end{aligned}
$$

Quite generally, once the QP self-energy is known, the QP excitation energy $\delta \mathcal{E}_{\mathrm{QP}}(\mathbf{k})$, which is the QP energy measured from the chemical potential $\mu$ of the interacting $\mathrm{EG}$, can be calculated by solving self-consistently the Dyson equation

$$
\delta \mathcal{E}_{\mathrm{QP}}(\mathbf{k})=\xi_{\mathbf{k}}+\left.\operatorname{Re} \Sigma_{\text {ret }}^{\mathrm{R}}(\mathbf{k}, \omega)\right|_{\omega=\delta \mathcal{E}_{\mathrm{QP}}(\mathbf{k}) / \hbar},
$$

where $\operatorname{Re} \Sigma_{\text {ret }}^{\mathrm{R}}(\mathbf{k}, \omega)=\operatorname{Re} \Sigma_{\text {ret }}(\mathbf{k}, \omega)-\Sigma_{\text {ret }}\left(k_{F}, 0\right)$. For later purposes we introduce at this point the so-called on-shell approximation (OSA). This amounts to approximating the QP excitation energy by calculating $\operatorname{Re} \sum_{\text {ret }}^{\mathrm{R}}(\mathbf{k}, \omega)$ in Eq. (9) at the frequency $\omega=\xi_{\mathbf{k}} / \hbar$ corresponding to the single-particle energy; that is,

$$
\delta \mathcal{E}_{\mathrm{QP}}(\mathbf{k}) \simeq \xi_{\mathbf{k}}+\left.\operatorname{Re} \sum_{\mathrm{ret}}^{\mathrm{R}}(\mathbf{k}, \omega)\right|_{\omega=\xi_{\mathbf{k}} / \hbar} .
$$

We will provide next the necessary background for a formal justification of Eqs. (2) and (3). The validity of the theory rests on the results of two completely different methods: diagrammatic perturbation theory (Sec. II B) and a renormalized Hamiltonian approach (Sec. II C).

\section{B. Theoretical foundations I: Diagrammatic perturbation theory}

In this section we make use a diagrammatic approach first developed by $\mathrm{Ng}$ and Singwi, ${ }^{34}$ and built on earlier ideas by Vignale and Singwi. ${ }^{25}$ The starting point is the exact identity ${ }^{3,59}$

$$
\delta \Sigma_{\sigma}(\mathbf{k}, \omega)=i \sum_{\sigma^{\prime}} \int \frac{d^{2} \mathbf{k}^{\prime} d \omega^{\prime}}{(2 \pi)^{3}} I_{\mathbf{k} \omega \sigma, \mathbf{k}^{\prime} \omega^{\prime} \sigma^{\prime}}(0) \delta G_{\sigma^{\prime}}\left(\mathbf{k}^{\prime}, \omega^{\prime}\right),
$$

where $\delta \Sigma_{\sigma}(\mathbf{k}, \omega)$ and $\delta G_{\sigma}(\mathbf{k}, \omega)$ are infinitesimal changes in the self-energy and the Green's function, and $I_{\mathbf{k} \omega \sigma, \mathbf{k}^{\prime} \omega^{\prime} \sigma^{\prime}}(0)$ is the irreducible electron-hole interaction at zero momentum and energy transfer. This identity is graphically represented in Fig. 1. The defining feature of the irreducible electronhole scattering block $I$ is that it includes only diagrams that cannot be divided into two parts by cutting a single electronhole pair propagator carrying zero energy and momentum.

The differential relation (11) cannot be integrated as it stands because $I$ is a complicated functional of $G$. The idea of $\mathrm{Ng}$ and Singwi was to use an approximate form of

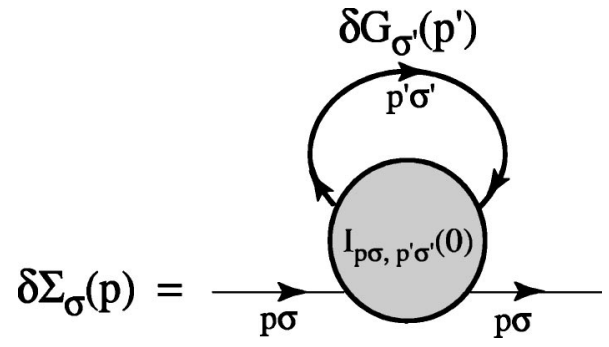

FIG. 1. Diagrammatic representation of the identity (11). Here and in the following we use the four-momentum variable $p$ as a shorthand for $(\mathbf{k}, \omega)$.

$I_{\mathbf{k} \omega \sigma, \mathbf{k}^{\prime} \omega^{\prime} \sigma^{\prime}}(0)$ that does not depend on $G$. The "local approximation" introduced by Vignale and Singwi in their study of the effective electron-electron interaction ${ }^{25}$ is useful for this purpose because it yields by physical arguments an expression of the form

$$
I_{\mathbf{k} \omega \sigma, \mathbf{k}^{\prime} \omega^{\prime} \sigma^{\prime}}(0) \simeq V_{\sigma \sigma^{\prime}}^{\mathrm{eff}}\left(\mathbf{k}-\mathbf{k}^{\prime}, \omega-\omega^{\prime}\right),
$$

where $V_{\sigma \sigma^{\prime}}^{\text {eff }}$ is just a function of the momentum and energy transfers in the electron-hole channel. Thus the main characteristic of the Ng-Singwi approach is that the key approximation in Eq. (12) is made on the irreducible electron-hole interaction rather than on the self-energy itself. With this approximation we can integrate Eq. (11) and obtain, up to an integration constant, the result

$$
\Sigma_{\sigma}(\mathbf{k}, \omega)=i \sum_{\sigma^{\prime}} \int \frac{d^{2} \mathbf{k}^{\prime} d \omega^{\prime}}{(2 \pi)^{3}} V_{\sigma \sigma^{\prime}}^{\mathrm{eff}}\left(\mathbf{k}-\mathbf{k}^{\prime}, \omega-\omega^{\prime}\right) G_{\sigma^{\prime}}\left(\mathbf{k}^{\prime}, \omega^{\prime}\right) .
$$

With the replacements $\mathbf{k}-\mathbf{k}^{\prime}=\mathbf{q}$ and $\omega-\omega^{\prime}=\Omega$, this expression has the form of the so-called GW approximation ${ }^{60}$ except for two crucial differences: (i) the effective interaction $V_{\sigma \sigma^{\prime}}^{\mathrm{eff}}(\mathbf{q}, \Omega)$ includes vertex corrections and is therefore more general than the screened interaction $W(\mathbf{q}, \Omega)$ between test charges that appears in the GW approximation; and (ii) the expression (13) involves an undetermined integration constant that must be fixed by independent means. For example, one can require $\Sigma\left(k_{F}, 0\right)$ to reproduce the correct value of the chemical potential as determined from QMC data. An analytic continuation procedure allows one to recast the timeordered self-energy in Eq. (13) into a retarded self-energy given by the sum of SX and $\mathrm{CH}$ contributions as in Eq. (1).

In their derivation of the local approximation in Eq. (12), $\mathrm{Ng}$ and Singwi ${ }^{34}$ as well as Singwi and Vignale ${ }^{25}$ sorted the diagrams that contribute to the irreducible electron-hole interaction into a number of classes identified by characteristic graphical criteria of irreducibility. The theory becomes in this case "local" when the various irreducible blocks are assumed to depend only on the net wave vector of the electronhole propagators that connect them. The irreducible blocks are then expressed in terms of the appropriate many-body local fields. At this point, an important physical and consistency requirement of the diagrammatic analysis is that the very same assumptions (in particular, that on the wave-vector dependence), when applied to the corresponding calculations 


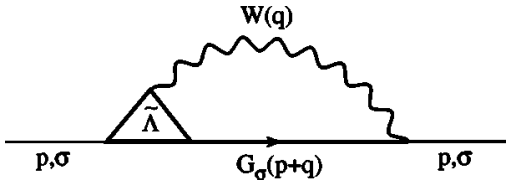

FIG. 2. Diagrammatic representation of Eq. (15). Here $W(\mathbf{q}, \Omega)=v_{\mathbf{q}} / \epsilon(\mathbf{q}, \Omega)$ is the usual test-charge-test-charge screened interaction.

of the charge- and spin-response functions lead to Eqs. (5) and (6). These approximations allow one to derive the following analytic expression for the effective interaction:

$$
\begin{gathered}
V_{\uparrow \uparrow}^{\mathrm{eff}}(\mathbf{q}, \Omega)=v_{\mathbf{q}}+\left\{v_{\mathbf{q}}\left[1-G_{+}(\mathbf{q})\right]\right\}^{2} \chi_{C}(\mathbf{q}, \Omega) \\
+\left[v_{\mathbf{q}} G_{-}(\mathbf{q})\right]^{2} \chi_{S}(\mathbf{q}, \Omega), \\
V_{\uparrow \downarrow}^{\mathrm{eff}}(\mathbf{q}, \Omega)=2\left[v_{\mathbf{q}} G_{-}(\mathbf{q})\right]^{2} \chi_{S}(\mathbf{q}, \Omega) .
\end{gathered}
$$

Inserting this into Eq. (13) and repeating standard analytical transformations, ${ }^{60}$ one easily recovers the expressions (2) and (3) for the screened-exchange and Coulomb-hole contributions to the self-energy given above.

Let us emphasize again that the result just obtained by the diagrammatic method rests on Eq. (13) for the self-energy (modulo an additive constant) and on the use of the effective interaction of Eq. (14). It can be readily seen from the analyses of Refs. 25 and 34 that no diagrams for $I_{\mathbf{k} \omega \sigma, \mathbf{k}^{\prime} \omega^{\prime} \sigma^{\prime}}(0)$ have been double counted. Rather, many diagrams have been dropped, the result for the self-energy eventually being adjusted a posteriori by fixing an additive constant imposing the correct value of the chemical potential. This must be contrasted with the results of a similar local analysis carried out, however, directly for the diagrammatic structure of the self-energy itself (as opposed to its variation). In this case one starts from the exact expression

$$
\begin{aligned}
\Sigma_{\sigma}(\mathbf{k}, \omega)= & i \sum_{\sigma^{\prime}} \int \frac{d^{2} \mathbf{k}^{\prime} d \omega^{\prime}}{(2 \pi)^{3}} \frac{v_{\mathbf{k}-\mathbf{k}^{\prime}}}{\epsilon\left(\mathbf{k}-\mathbf{k}^{\prime}, \omega-\omega^{\prime}\right)} \\
& \times \tilde{\Lambda}_{\mathbf{k} \omega, \mathbf{k}^{\prime} \omega^{\prime}} G_{\sigma^{\prime}}\left(\mathbf{k}^{\prime}, \omega^{\prime}\right),
\end{aligned}
$$

where $\tilde{\Lambda}$ is the proper vertex function and $\epsilon$ is the regular dielectric function (see Fig. 2). Within this local approximation one finds ${ }^{21,31,61}$

$$
\tilde{\Lambda}_{\mathbf{k} \omega, \mathbf{k}^{\prime} \omega^{\prime}}=\frac{1}{1+v_{\mathbf{k}-\mathbf{k}^{\prime}} G_{+}\left(\mathbf{k}-\mathbf{k}^{\prime}\right) \chi_{0}\left(\mathbf{k}-\mathbf{k}^{\prime}, \omega-\omega^{\prime}\right)},
$$

so that this route to the self-energy includes only the contribution of charge fluctuations but, unphysically, misses completely that of spin fluctuations. The root of the difficulty obviously lies in the fact that the local approximation for the vertex function is not good enough to capture the contribution of spin-density fluctuations. On the other hand, the dependence of $I$ on spin fluctuations is manifest in the terms proportional to $G_{-}^{2}$ in Eq. (14). This is the main physical reason why it is better to apply the local approximation to the differential relation (11) than to the integral relation (15). In fact, all quasiparticle properties of our present interest de- pend on relative variations of the self-energy, i.e., on $\delta \Sigma$ rather than on the absolute value of $\Sigma$.

\section{Theoretical foundations II: The renormalized Hamiltonian approach}

In this section we review the derivation of Eqs. (2) and (3) from the point of view of an effective renormalized Hamiltonian (the RHA referred to above) for the low-energy degrees of freedom of the electron liquid, a quantity that provides a vivid theoretical realization of the Landau quasiparticles. ${ }^{24,27,28}$ Details of this derivation can be found in the original papers or in Ref. 3.

One starts by dividing the Hilbert space of the original and well-known EG Hamiltonian $\mathcal{H}_{\mathrm{EG}}$ into a "slow" sector $\left(\mathcal{S}_{\Lambda}\right)$ and a "fast" sector $\left(\mathcal{F}_{\Lambda}\right)$, assuming the existence of the Fermi surface at $k=k_{F} . \mathcal{S}_{\Lambda}$, contains only plane-wave states with wave vector $\mathbf{k}$ close to the Fermi surface, i.e., such that $\left|k-k_{F}\right|<\Lambda$, where $\Lambda$ is an arbitrarily small cutoff. $\mathcal{F}_{\Lambda}$ contains all the other states. We correspondingly introduce slow and fast creation and annihilation operators, which operate in these two sectors,

$$
\hat{c}_{\mathbf{k}, \sigma}= \begin{cases}\hat{s}_{\mathbf{k}, \sigma}, & \mathbf{k} \in \mathcal{S}_{\Lambda} \\ \hat{f}_{\mathbf{k}, \sigma}, & \mathbf{k} \in \mathcal{F}_{\Lambda} .\end{cases}
$$

Our goal is to derive an effective Hamiltonian for the slow sector that contains only the $\hat{s}_{\mathbf{k}, \sigma}$ operators by integrating out in a reasoned manner the $\hat{f}_{\mathbf{k}, \sigma}$ degrees of freedom.

$\mathcal{H}_{\mathrm{EG}}$ is first rewritten using the $\hat{s}_{\mathbf{k}, \sigma}$ and $\hat{f}_{\mathbf{k}, \sigma}$ operators,

$$
\mathcal{H}_{\mathrm{EG}}=\mathcal{H}_{\mathrm{s}}+\mathcal{H}_{\mathrm{f}}+\mathcal{H}_{\mathrm{sf}}
$$

For instance, the first term reads

$$
\begin{aligned}
\mathcal{H}_{\mathrm{s}}= & \sum_{\mathbf{k}, \sigma} \varepsilon_{\mathbf{k}} \hat{s}_{\mathbf{k}, \sigma}^{\dagger} \hat{s}_{\mathbf{k}, \sigma}+\frac{1}{2 S} \sum_{\mathbf{q} \neq 0} v_{\mathbf{q}} \sum \sum_{\mathbf{k}_{1}, \sigma_{1}} \sum_{\mathbf{k}_{2}, \sigma_{2}} \\
& \times \hat{s}_{\mathbf{k}_{1}+\mathbf{q}, \sigma_{1}}^{\dagger} \hat{s}_{\mathbf{k}_{2}-\mathbf{q}, \sigma_{2}}^{\dagger} \hat{s}_{\mathbf{k}_{2}, \sigma_{2}} \hat{s}_{\mathbf{k}_{1}, \sigma_{1}},
\end{aligned}
$$

where all wave vectors belong to $\mathcal{S}_{\Lambda}$. $\mathcal{H}_{\mathrm{f}}$ has a similar structure and tends to the full EG Hamiltonian in the limit $\Lambda$ $\rightarrow 0$, a property that will turn useful in what follows.

The third term $\mathcal{H}_{\text {sf }}$ describes the interaction between the slow and fast particles; although this term is the sum of 14 different terms, we assume that the relevant operators are those that separately conserve the number of particles in the two sectors [i.e., terms of the type $\hat{s}_{\mathbf{k}_{1}+\mathbf{q}, \sigma_{1}}^{\dagger} \hat{s}_{\mathbf{k}_{2}-\mathbf{q}, \sigma_{2}}^{\dagger} \hat{s}_{\mathbf{k}_{2}, \sigma_{2}} \hat{f}_{\mathbf{k}_{1}, \sigma_{1}}$ (trilinear in the field operators of either slow or fast particles) or $\hat{s}_{\mathbf{k}_{1}+\mathbf{q}, \sigma_{1}}^{\dagger} \hat{s}_{\mathbf{k}_{2}-\mathbf{q}, \sigma_{2}}^{\dagger} \hat{f}_{\mathbf{k}_{2}, \sigma_{2}} \hat{f}_{\mathbf{k}_{1}, \sigma_{1}}$ will be dropped]. Four terms are left within this assumption. Accordingly, by simple algebraic manipulations $\mathcal{H}_{\text {sf }}$ can be written as 


$$
\begin{aligned}
\mathcal{H}_{\mathrm{sf}}= & \frac{1}{S} \sum_{\mathbf{q} \neq 0} \sum_{\mathbf{k}_{1}, \sigma_{1}} \sum_{\mathbf{k}_{2}, \sigma_{2}} v_{\mathbf{q}} \hat{S}_{\mathbf{k}_{1}+\mathbf{q}, \sigma_{1}}^{\dagger} \hat{f}_{\mathbf{k}_{2}-\mathbf{q}, \sigma_{2}}^{\dagger} \hat{f}_{\mathbf{k}_{2}, \sigma_{2}} \hat{s}_{\mathbf{k}_{1}, \sigma_{1}} \\
& -\frac{1}{S} \sum_{\mathbf{q} \neq 0} \sum \sum_{\mathbf{k}_{1}, \sigma_{1}} v_{\mathbf{k}_{2}, \sigma_{2}} v_{\mathbf{k}_{2}-\mathbf{k}_{1}-\mathbf{q}} \hat{s}_{\mathbf{k}_{1}+\mathbf{q}, \sigma_{1}}^{\dagger} \hat{f}_{\mathbf{k}_{2}-\mathbf{q}, \sigma_{2}}^{\dagger} \hat{f}_{\mathbf{k}_{2}, \sigma_{1}} \hat{s}_{\mathbf{k}_{1}, \sigma_{2}},
\end{aligned}
$$

where $\mathbf{k}_{1}$ and $\mathbf{k}_{1}+\mathbf{q}$ belong to $\mathcal{S}_{\Lambda}$, and $\mathbf{k}_{2}$ and $\mathbf{k}_{2}-\mathbf{q}$ belong to $\mathcal{F}_{\Lambda}$.

The first (direct) term in $\mathcal{H}_{\text {sf }}$ describes the Coulomb interaction between slow and fast particles and can then be expressed in terms of density fluctuations in the two sets of particles. The second (exchange) term describes an exchange process in which a slow particle replaces a fast particle and vice versa. Note that the two particles can have opposite spins (i.e., $\sigma_{1}=-\sigma_{2}$ ), and when this is the case a net spinangular momentum is exchanged between slow and fast particles. In practice this means that any attempt to write $\mathcal{H}_{\text {sf }}$ in terms of collective variables must also involve an interaction between slow and fast particles mediated by spin fluctuations.

All these arguments bring us to a second crucial approximation: we treat $\mathcal{H}_{\text {sf }}$ in an average sense by writing these microscopic processes in terms of interactions between density and spin-density fluctuations in the two sets of slow and fast particles,

$$
\begin{aligned}
\mathcal{H}_{\mathrm{sf}} \simeq & \frac{1}{S} \sum_{\mathbf{q} \neq 0} v_{\mathrm{C}}(\mathbf{q}) \sum_{\mathbf{k}, \sigma_{1}} \hat{n}_{-\mathbf{q}} \hat{s}_{\mathbf{k}-\mathbf{q}, \sigma_{1}}^{\dagger} \hat{s}_{\mathbf{k}, \sigma_{1}} \\
& +\frac{1}{S} \sum_{\mathbf{q} \neq 0} v_{\mathrm{S}}(\mathbf{q}) \sum_{\mathbf{k}, \sigma_{1}, \sigma_{2}} \hat{\mathbf{S}}_{-\mathbf{q}} \cdot\left(\hat{s}_{\mathbf{k}-\mathbf{q}, \sigma_{1}}^{\dagger}[\boldsymbol{\sigma}]_{\sigma_{1} \sigma_{2}} \hat{s}_{\mathbf{k}, \sigma_{2}}\right),
\end{aligned}
$$

where $\hat{n}_{\mathbf{q}}=\sum_{\mathbf{k}, \sigma} \hat{f}_{\mathbf{k}-\mathbf{q}, \sigma}^{\dagger} \hat{f}_{\mathbf{k}, \sigma}$ and $\hat{\mathbf{S}}_{\mathbf{q}}=\sum_{\mathbf{k}, \sigma, \sigma^{\prime}} \hat{f}_{\mathbf{k}-\mathbf{q}, \sigma}^{\dagger}[\boldsymbol{\sigma}]_{\sigma \sigma^{\prime}} \hat{f}_{\mathbf{k}, \sigma^{\prime}}$ are, respectively, the density and spin-density operators for the fast sector. The effective interaction potentials $v_{\mathrm{C}}(\mathbf{q})$ and $v_{S}(\mathbf{q})$ must include both the exchange and the correlation effects. This requirement can be fulfilled in an approximate way by means of local field factors $G_{+}(\mathbf{q})$ and $G_{-}(\mathbf{q})$ by taking $v_{\mathrm{C}}(\mathbf{q})=v_{\mathbf{q}}\left[1-G_{+}(\mathbf{q})\right]$ and $v_{\mathrm{S}}(\mathbf{q})=-v_{\mathbf{q}} G_{-}(\mathbf{q})$. Note that, as in Eq. (14), we are again using frequency-independent local field factors for the reasons indicated in Sec. III.

We now carry out a unitary transformation that eliminates the interaction $\mathcal{H}_{\mathrm{sf}}$ between slow and fast particles to first order in its strength. ${ }^{30}$ We search for a Hermitian operator $\hat{\Theta}_{\Lambda}$, which maps the original Hamiltonian into a new Hamiltonian $\mathcal{H}^{\prime}=\exp \left(i \hat{\Theta}_{\Lambda}\right) \mathcal{H}_{\mathrm{EG}} \exp \left(-i \hat{\Theta}_{\Lambda}\right)$ having the same eigenvalues but transformed eigenfunctions. The generator $\hat{\Theta}_{\Lambda}$ is at least of first order in the strength of the interaction between the two sectors. Accordingly just expanding in powers of $\hat{\Theta}_{\Lambda}$ the expression for $\mathcal{H}^{\prime}$, one sees that the interaction term $\mathcal{H}_{\text {sf }}$ is eliminated to first order by choosing $\hat{\Theta}_{\Lambda}$ as the solution of the operatorial equation

$$
i\left[\hat{\Theta}_{\Lambda}, \mathcal{H}_{\mathrm{s}}+\mathcal{H}_{\mathrm{f}}\right]=-\mathcal{H}_{\mathrm{sf}}
$$

We finally obtain the effective Hamiltonian for the lowenergy degrees of freedom of the electron liquid by averaging over the ground state $|0\rangle$ of the Hamiltonian $\mathcal{H}_{\mathrm{f}}$. We have

$$
\mathcal{H}_{\mathrm{QP}} \equiv\left\langle 0\left|\mathcal{H}^{\prime}\right| 0\right\rangle=E_{0}+\mathcal{H}_{\mathrm{s}}+\frac{i}{2}\left\langle 0\left|\left[\hat{\Theta}_{\Lambda}, \mathcal{H}_{\mathrm{sf}}\right]\right| 0\right\rangle .
$$

Obviously the constant $E_{0}$ does not play any physical role and will be dropped from now on.

The operatorial equation (22) can be solved for $\hat{\Theta}_{\Lambda}$ once the commutator of $\hat{\Theta}_{\Lambda}$ with the interaction term in $\mathcal{H}_{\mathrm{s}}$ is dropped, the justification being that this commutator vanishes upon averaging on the ground state of the fast sector. A lengthy but straightforward calculation yields

$$
\begin{aligned}
\mathcal{H}_{\mathrm{QP}}= & \sum_{\mathbf{k}, \sigma}\left[\varepsilon_{\mathbf{k}}-\frac{1}{S} \sum_{\mathbf{q}} \int_{0}^{+\infty} \frac{d \omega^{\prime}}{\pi} \frac{v_{\mathrm{C}}^{2}(\mathbf{q}) \operatorname{Im} \chi_{\mathrm{C}}\left(\mathbf{q}, \omega^{\prime}\right)+3 v_{\mathrm{S}}^{2}(\mathbf{q}) \operatorname{Im} \chi_{\mathrm{S}}\left(\mathbf{q}, \omega^{\prime}\right)}{\Delta_{\mathbf{k}, \mathbf{q}}-\omega^{\prime}}\right] \hat{s}_{\mathbf{k}, \sigma}^{\dagger} \hat{s}_{\mathbf{k}, \sigma} \\
& +\frac{1}{2 S} \sum_{\mathbf{q} \neq 0}\left[v_{\mathbf{q}}+v_{\mathrm{C}}^{2}(\mathbf{q}) \chi_{\mathrm{C}}\left(\mathbf{q}, \Delta_{\mathbf{k}, \mathbf{q}}\right)\right] \sum_{\mathbf{k}_{1}, \sigma_{1}} \sum_{\mathbf{k}_{2}, \sigma_{2}} \hat{s}_{\mathbf{k}_{1}+\mathbf{q}, \sigma_{1}}^{\dagger} \hat{s}_{\mathbf{k}_{2}-\mathbf{q}, \sigma_{2}}^{\dagger} \hat{s}_{\mathbf{k}_{2}, \sigma_{2}} \hat{s}_{\mathbf{k}_{1}, \sigma_{1}} \\
& +\frac{1}{2 S} \sum_{\mathbf{q} \neq 0} v_{\mathrm{S}}^{2}(\mathbf{q}) \chi_{\mathrm{S}}\left(\mathbf{q}, \Delta_{\mathbf{k}, \mathbf{q}}\right) \sum \sum_{\mathbf{k}_{1}, \sigma_{1}} \sum_{\mathbf{k}_{2}, \sigma_{2}} \sum_{\tau_{1}, \tau_{2}}[\boldsymbol{\sigma}]_{\sigma_{1} \sigma_{2}} \cdot[\boldsymbol{\sigma}]_{\tau_{1} \tau_{2}} \hat{s}_{\mathbf{k}_{1}+\mathbf{q}, \sigma_{1}}^{\dagger} \hat{s}_{\mathbf{k}_{2}-\mathbf{q}, \tau_{1}}^{\dagger} \hat{s}_{\mathbf{k}_{2}, \tau_{2}} \hat{s}_{\mathbf{k}_{1}, \sigma_{2}},
\end{aligned}
$$

where $\Delta_{\mathbf{k}, \mathbf{q}}=\left(\varepsilon_{\mathbf{k}}-\varepsilon_{\mathbf{k}-\mathbf{q}}\right) / \hbar$. Note that the sums over the eigenstates of $\mathcal{H}_{\mathrm{f}}$ appearing in the derivation have been carried out using the identity

$$
\frac{1}{S} \sum_{n} \frac{\left|\left\langle n\left|\hat{n}_{\mathbf{q}}\right| 0\right\rangle\right|^{2}}{\varepsilon_{\mathbf{k}}-\varepsilon_{\mathbf{k}-\mathbf{q}}-\left(E_{n}-E_{0}\right)}=-\frac{1}{\pi} \int_{0}^{+\infty} d \omega^{\prime} \frac{\operatorname{Im} \chi_{\mathrm{C}}\left(\mathbf{q}, \omega^{\prime}\right)}{\left(\varepsilon_{\mathbf{k}}-\varepsilon_{\mathbf{k}-\mathbf{q}}\right) / \hbar-\omega^{\prime}} .
$$

A similar identity for $\hat{S}_{z, \mathbf{q}}$ has also been employed. Here $\chi_{\mathrm{C}}(\mathbf{q}, \omega)$ and $\chi_{\mathrm{S}}(\mathbf{q}, \omega)$ are the response functions of the "medium" (the fast degrees of freedom), which asymptotically tend to those of the full EG for $\Lambda \rightarrow 0$. 
The QP Hamiltonian $\mathcal{H}_{\mathrm{QP}}$ as written in Eq. (24) has a very clear physical meaning. It is the Hamiltonian for a gas of weakly interacting slow-moving particles with a singleparticle dispersion relation shifted by a self-interaction term (the so-called Coulomb-hole shift). The reason for the gas being weakly interacting is that the quartic term in $\mathcal{H}_{\mathrm{QP}}$ tends to zero faster than the kinetic-energy term in the limit $\Lambda$ $\rightarrow 0$. We can thus calculate the quasiparticle energy within first-order perturbation theory, with the result

$$
\mathcal{E}_{\mathrm{QP}}(\mathbf{k})=\varepsilon_{\mathbf{k}}+E_{\mathrm{SX}}(\mathbf{k})+E_{\mathrm{CH}}(\mathbf{k}) .
$$

Note that $\mathcal{E}_{\mathrm{SX}}(\mathbf{k})$ is here a self-energy calculated with a dynamically screened effective interaction [the quartic part of Eq. (24), which is equivalent to the Kukkonen-Overhauser QP-QP interaction],

$$
\begin{aligned}
\mathcal{E}_{\mathrm{SX}}(\mathbf{k})= & -\int \frac{d^{2} \mathbf{q}}{(2 \pi)^{2}}\left[v_{\mathbf{q}}+v_{\mathrm{C}}^{2}(\mathbf{q}) \chi_{\mathrm{C}}\left(\mathbf{q}, \Delta_{\mathbf{k}, \mathbf{q}}\right)\right. \\
& \left.+3 v_{\mathrm{S}}^{2}(\mathbf{q}) \chi_{\mathrm{S}}\left(\mathbf{q}, \Delta_{\mathbf{k}, \mathbf{q}}\right)\right] \Theta\left(-\xi_{\mathbf{k}-\mathbf{q}} / \hbar\right) .
\end{aligned}
$$

The last term in Eq. (26) is the Coulomb shift, which can be seen as being generated by normal ordering with respect to the vacuum,

$$
\begin{aligned}
\mathcal{E}_{\mathrm{CH}}(\mathbf{k})= & -\int \frac{d^{2} \mathbf{q}}{(2 \pi)^{2}} \int_{0}^{+\infty} \frac{d \omega^{\prime}}{\pi} \\
& \times \frac{v_{\mathrm{C}}^{2}(\mathbf{q}) \operatorname{Im} \chi_{\mathrm{C}}\left(\mathbf{q}, \omega^{\prime}\right)+3 v_{\mathrm{S}}^{2}(\mathbf{q}) \operatorname{Im} \chi_{\mathrm{S}}\left(\mathbf{q}, \omega^{\prime}\right)}{\Delta_{\mathbf{k}, \mathbf{q}}-\omega^{\prime}} .
\end{aligned}
$$

The sum of $\mathcal{E}_{\mathrm{SX}}(\mathbf{k})$ and $\mathcal{E}_{\mathrm{CH}}(\mathbf{k})$ coincides with Eq. (7), calculated at the single-particle frequency $\omega=\xi_{\mathbf{k}} / \hbar$.

\section{LOCAL-FIELD FACTORS}

As is clear from Eqs. (1)-(8), the local-field factors are fundamental quantities for an evaluation of quasiparticle properties. In this section we introduce the static values of these functions that we have chosen to calculate the real and imaginary parts of the QP self-energy from Eqs. (7) and (8). Although the local-field factors are frequency dependent quantities in what follows, we shall make the common, and to a certain extent uncontrolled, approximation of neglecting their frequency dependence. Recent studies ${ }^{62}$ have explored such a dependence in the long-wavelength limit $\mathbf{q} \rightarrow 0$, but clearly the knowledge of the full dependence on wave number is necessary for correctly carrying out the type of calculations in which we are interested.

Analytical expressions for the relevant static local-field factors $G_{+}(\mathbf{q})$ and $G_{-}(\mathbf{q})$, are available, ${ }^{35}$ which reproduce the most recent diffusion Monte Carlo data ${ }^{10,15}$ and, as we are going to summarize below, embody the exact asymptotic behaviors at both small and large wave numbers $q$. Specifically, in the long wavelength limit our choice satisfies the compressibility and spin-susceptibility sum rules,

$$
\lim _{q \rightarrow 0} G_{ \pm}(\mathbf{q})=A_{ \pm} \frac{q}{k_{F}}
$$

with $A_{+}=\left(1-\kappa_{0} / \kappa\right) /\left(r_{S} \sqrt{2}\right)$ and $A_{-}=\left(1-\chi_{P} / \chi_{S}\right) /\left(r_{s} \sqrt{2}\right)$. Here $\kappa_{0}$ is the compressibility of the noninteracting gas, $\kappa$ and $\chi_{S}$ are the compressibility and the spin susceptibility of the interacting system, and $\chi_{P}$ is the Pauli spin susceptibility. By making use of the thermodynamic definitions of $\kappa$ and $\chi_{S}$ we can write

$$
\frac{\kappa_{0}}{\kappa}=1-\frac{\sqrt{2}}{\pi} r_{s}+\frac{r_{s}^{4}}{8}\left[\frac{\partial^{2} \varepsilon_{c}\left(r_{s}, 0\right)}{\partial r_{s}^{2}}-\frac{1}{r_{s}} \frac{\partial \varepsilon_{c}\left(r_{s}, 0\right)}{\partial r_{s}}\right],
$$

and

$$
\frac{\chi_{P}}{\chi_{S}}=1-\frac{\sqrt{2}}{\pi} r_{s}+\left.\frac{r_{s}^{2}}{2} \frac{\partial^{2} \varepsilon_{c}\left(r_{s}, \zeta\right)}{\partial \zeta^{2}}\right|_{\zeta=0},
$$

where $\varepsilon_{c}\left(r_{s}, \zeta\right)$ is the correlation energy per particle as a function of $r_{s}$ and of the degree $\zeta$ of spin polarization. ${ }^{13,18}$

At large $q$, on the other hand, the local fields of Ref. 35 satisfy the asymptotic behavior ${ }^{63,64}$

$$
G_{ \pm}(\mathbf{q}) \rightarrow C \frac{q}{k_{F}}+B_{ \pm} .
$$

Here $C$ is determined by the difference in kinetic energy between the interacting and the ideal Fermi gas,

$$
C=-\frac{r_{s}}{2 \sqrt{2}} \frac{\partial}{\partial r_{s}}\left[r_{s} \varepsilon_{c}\left(r_{s}, 0\right)\right],
$$

while $B_{+}=1-g(0)$ and $B_{-}=g(0)$, with $g(0)$ being the value of the pair-distribution function at the origin. ${ }^{65}$

\section{NUMERICAL RESULTS}

We turn to a presentation of our main numerical results. In Sec. IV A we present some illustrative results for the QP excitation energy and lifetime, and in Section IV B we give our results for the QP effective mass and renormalization constant. In all figures the labels "RPA," " $G_{+}$," and " $G_{+} \& G_{-}$" refer to three possible choices for the local-field factors. "RPA" refers to the case in which local-field factors are not included, " $G_{+}$" to the case in which the antisymmetric spin-spin local field is set to zero (i.e., spin-density fluctuations are not allowed); and, finally, " $G_{+} \& G_{-}$" refers to the full theory including both charge- and spin-density fluctuations.

\section{A. Quasiparticle self-energy}

We have computed the real and imaginary parts of the QP self-energy using Eqs. (7) and (8). In Figs. 3 and 4 we show the real part of the SX and $\mathrm{CH}$ contributions as from Eqs. (2) and (3), evaluated at the single-particle frequency $\omega=\xi_{\mathbf{k}} / \hbar$ and measured from their value at $k=k_{F}$. Note the presence of a strong dip in the $\mathrm{CH}$ term at a value of $k$ ( $k_{p}$, for example) which depends on $r_{s}$ and on the functional form of the charge-charge local-field factor. This is the plasmon dip, which is also present in three dimensions and originates from 

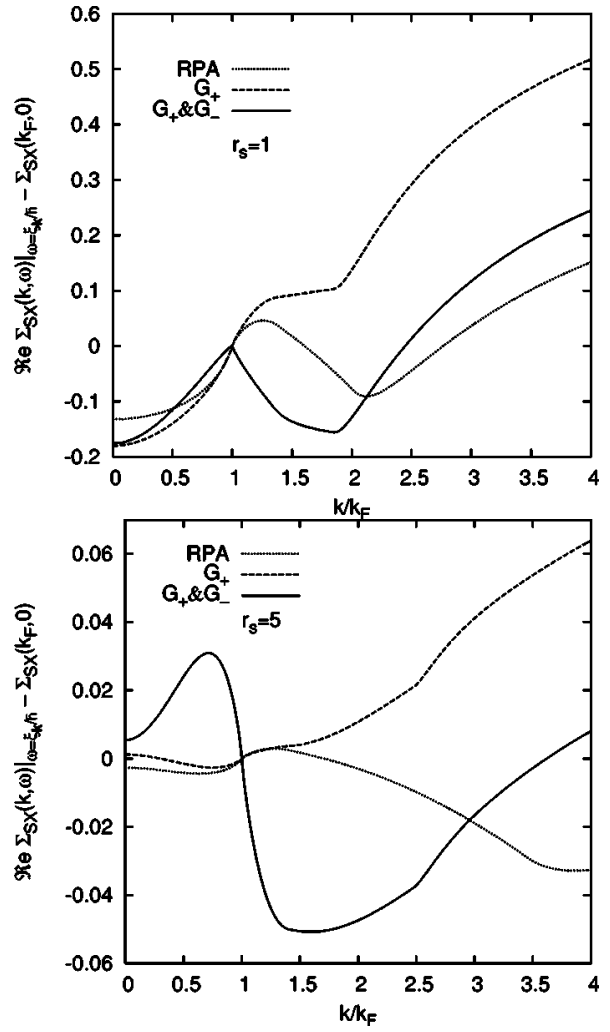

FIG. 3. The real part of the SX contribution to the retarded self-energy (in units of Ry) evaluated at $\omega=\xi_{\mathbf{k}} / \hbar$ as a function of $k / k_{F}$ for $r_{s}=1$ (top panel) and 5 (bottom panel).

the fact that at each $r_{s}$ there is a sufficiently high value of $k$ for decay of an electron-hole pair into a plasmon with conservation of momentum and energy. Mathematically this dip arises for the reasons explained in Appendix A.

In Fig. 5 we show $\operatorname{Re} \Sigma_{\text {ret }}^{R}(\mathbf{k}, \omega)$ as from Eq. (7), evaluated at $\omega=\xi_{\mathbf{k}} / \hbar$. There is substantial cancellation between the SX and $\mathrm{CH}$ contributions for $k<k_{F}$, so that in this range the QP self-energy is essentially very weakly momentum dependent. ${ }^{5}$ Such a function has a Fourier transform, which is to a good extent local in real space, and this result can be viewed as a microscopic justification of the local-density approximation to the exchange-correlation potential of densityfunctional theory.

In Fig. 6 we show the absolute value of $\operatorname{Im} \Sigma_{\text {ret }}(\mathbf{k}, \omega)$ as from Eq. (8), evaluated at $\omega=\xi_{\mathbf{k}} / \hbar$. This function takes a finite jump at the wave number of the plasmon dip. The discontinuity is peculiar to two dimensions: ${ }^{66}$ it is absent in three dimensions and arises from the fact that the oscillator strength of the plasmon pole is nonzero at $k_{p}$ (see Appendix A). The qualitative difference in the shape of the imaginary part of the self-energy below and above $k_{p}$ reflects the opening of a new decay channel for an electron-hole pair.

\section{B. Many-body effective mass enhancement}

Once the QP excitation energy is known, the effective mass $m^{*}$ can be calculated by means of the relationship
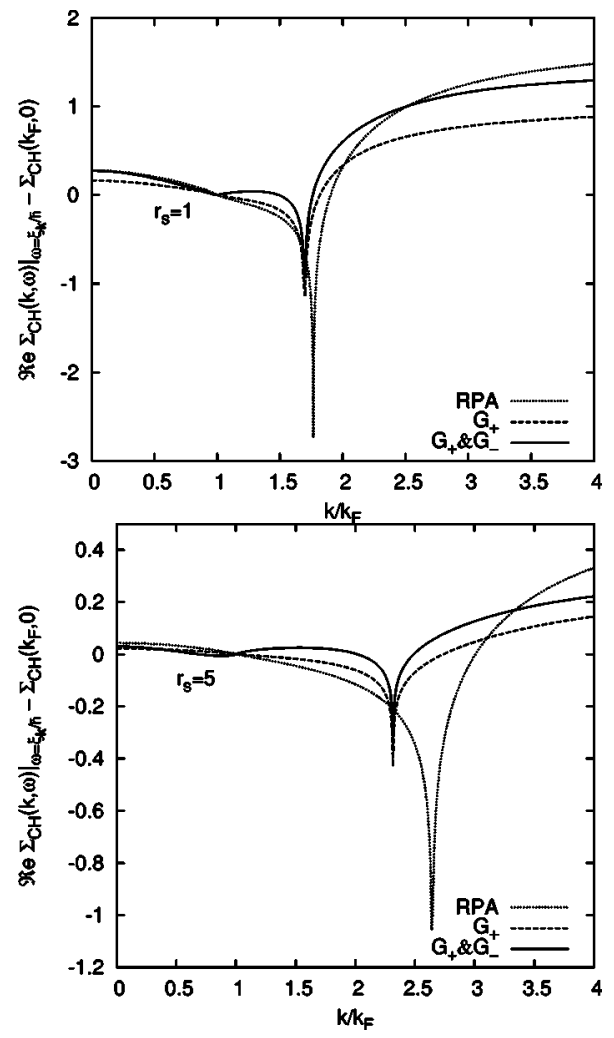

FIG. 4. The real part of the $\mathrm{CH}$ contribution to the retarded self-energy (in units of Ry) evaluated at $\omega=\xi_{\mathbf{k}} / \hbar$ as a function of $k / k_{F}$ for $r_{s}=1$ (top panel) and 5 (bottom panel).

$$
\frac{1}{m^{*}}=\left.\frac{1}{\hbar^{2} k_{F}} \frac{d \delta \mathcal{E}_{\mathrm{QP}}(k)}{d k}\right|_{k=k_{F}} .
$$

In Sec. II we remarked that the QP excitation energy may be calculated either by solving self-consistently the Dyson equation (9) or by using the OSA in Eq. (10). In what follows the identity

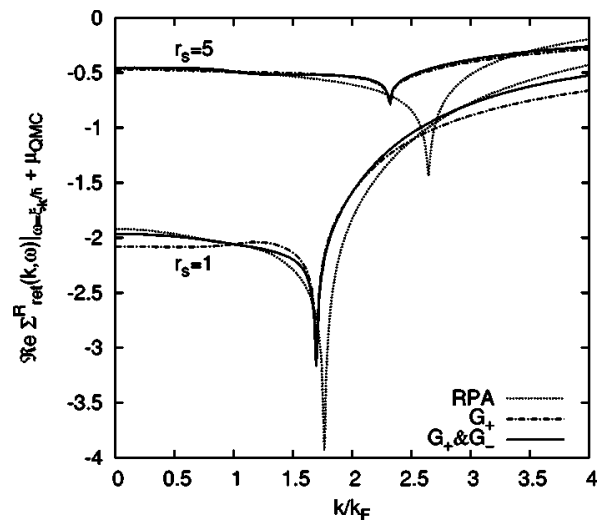

FIG. 5. The real part of the retarded self-energy (in units of Ry) evaluated at $\omega=\xi_{\mathbf{k}} / \hbar$ as a function of $k / k_{F}$ for $r_{s}=1$ and 5. $\mu_{\mathrm{QMC}}$ is the chemical potential from the QMC ground-state energy. 


$$
\begin{aligned}
\frac{d \operatorname{Re} \sum_{\text {ret }}^{\mathrm{R}}[k, \omega(k)]}{d k}= & \left.\partial_{k} \operatorname{Re} \Sigma_{\mathrm{ret}}^{\mathrm{R}}(k, \omega)\right|_{\omega=\omega(k)} \\
& +\left.\partial_{\omega} \operatorname{Re} \Sigma_{\mathrm{ret}}^{\mathrm{R}}(k, \omega)\right|_{\omega=\omega(k)} \frac{d \omega(k)}{d k}
\end{aligned}
$$

will be used, $\omega(k)$ being an arbitrary function of $k$.

Using Eqs. (34) and (35) with $\omega(k)=\delta \mathcal{E}_{\mathrm{QP}}(k) / \hbar$ we find that the effective mass $m_{\mathrm{D}}^{*}$ calculated within the Dyson scheme is given by

$$
\frac{m_{\mathrm{D}}^{*}}{m}=\frac{Z^{-1}}{1+\left.\left(m / \hbar^{2} k_{F}\right) \partial_{k} \operatorname{Re} \sum_{\mathrm{ret}}^{\mathrm{R}}(k, \omega)\right|_{k=k_{F}, \omega=0}} .
$$

The renormalization constant $Z$ that measures the discontinuity of the momentum distribution at $k=k_{F}$ is given by

$$
Z=\frac{1}{1-\left.\hbar^{-1} \partial_{\omega} \operatorname{Re} \Sigma_{\text {ret }}^{\mathrm{R}}(k, \omega)\right|_{k=k_{F}, \omega=0}} .
$$

The normal Fermi-liquid assumption, $0<Z \leqslant 1$, implies $\left.\partial_{\omega} \operatorname{Re} \sum_{\text {ret }}^{\mathrm{R}}(k, \omega)\right|_{k=k_{F}, \omega=0} \leqslant 0$. Thus we see that the effective mass $m_{\mathrm{D}}^{*}$ can diverge at a finite value of $r_{s}$ by one of two mechanisms: (i) the partial derivative of $\sum_{\text {ret }}^{\mathrm{R}^{s}}$ with respect to $\omega,\left.\quad \partial_{\omega} \operatorname{Re} \sum_{\text {ret }}^{\mathrm{R}}(k, \omega)\right|_{k=k_{F}, \omega=0}$ going to minus infinity at some finite value of $r_{s}$, ${ }^{67}$ (ii) the partial derivative of $\sum_{\text {ret }}^{\mathrm{R}}$ with respect to $k,\left.\quad \partial_{\omega} \operatorname{Re} \sum_{\text {ret }}^{\mathrm{R}}(k, \omega)\right|_{k=k_{F}, \omega=0}$ going to $-\hbar^{2} k_{F} / m$ at some finite value of $r_{s}$. Neither possibility is realized in our calculation: the first is barred a priori by the fact that the analytic expression for the frequency derivative of $\sum_{\text {ret }}^{R}$ is always finite at finite $r_{s}$; the second is found a posteriori not to occur since the momentum derivative of $\Sigma_{\text {ret }}^{\mathrm{R}}$ is positive up to the largest $r_{s}$ considered (see below).

On the other hand, using Eqs. (34) and (35) and with $\omega(k)=\xi_{\mathbf{k}} / \hbar$ we find that the effective mass $m_{\mathrm{OSA}}^{*}$ within the OSA is given by

$$
\frac{m_{\mathrm{OSA}}^{*}}{m}=\frac{1}{1+\left.\left(m / \hbar^{2} k_{F}\right) \partial_{k} \operatorname{Re} \Sigma_{\mathrm{ret}}^{\mathrm{R}}(k, \omega)\right|_{k=k_{F}, \omega=0}+\left.\hbar^{-1} \partial_{\omega} \operatorname{Re} \Sigma_{\mathrm{ret}}^{\mathrm{R}}(k, \omega)\right|_{k=k_{F}, \omega=0}} .
$$

Of course, Eq. (38) is a valid approximation to the effective mass in the weak coupling limit, as can be seen by expanding Eq. (36) for small values of $\Sigma_{\text {ret }}^{R}$, however, its application becomes problematic at large values of $r_{s}$. In particular, we see that because $-\partial_{\omega} \operatorname{Re} \sum_{\text {ret }}^{\mathrm{R}}\left(k_{F}, 0\right)$ increases monotonically with increasing $r_{s}$, there must necessarily be a critical value of $r_{s}$ for which the denominator of Eq. (38) vanishes and $m_{\text {OSA }}^{*}$ diverges. A recent paper by Zhang and Das Sarma ${ }^{68}$ infers from this fact a true divergence of the effective mass within the RPA. In our view, however, this must be considered an artifact of Eq. (38). Its unphysical character is revealed by the fact that the divergence is driven by a negative but finite value of $\partial_{\omega} \operatorname{Re} \Sigma_{\text {ret }}^{\mathrm{R}}\left(k_{F}, 0\right)$, whereas we know, from the general analysis, that a genuine divergence would have to be driven either by an infinite $\partial_{\omega} \operatorname{Re} \Sigma_{\text {ret }}^{\mathrm{R}}\left(k_{F}, 0\right)$ or by a negative $\partial_{k} \operatorname{Re} \sum_{\text {ret }}^{\mathrm{R}}\left(k_{F}, 0\right)$ becoming equal to $-\hbar^{2} k_{F} / m$. We conclude that there is no evidence, within the present theory, for a divergence of the effective mass.

In Fig. 7 we show our numerical results for $m_{\mathrm{D}}^{*}$ and $m_{\mathrm{OSA}}^{*}$. The effective mass enhancement is substantially smaller in the Dyson-equation calculation than in the OSA, the reason being that a large cancellation occurs between the numerator and denominator in Eq. (36). In both calculations the combined effect of charge and spin fluctuations is to enhance the effective mass over the RPA result, whereas the opposite effect is found if only charge fluctuations are included-a manifestly incorrect result that neglects the spinorial nature of the electron. For completeness we have also included in Fig. 7 the variational QMC results of Kwon et al. ${ }^{11}$ The reader should bear in mind that the effective mass is not a ground-state property and thus its evaluation by the QMC technique is quite delicate, as it involves the construction of excited states. There clearly is quantitative disagreement between our "best" theoretical results (the $G_{+} \& G_{-} / \mathrm{D}$ predictions) and the QMC data.

In Fig. 8 we show the behavior of the two terms in the denominator of Eq. (38) as functions of $r_{s}$. This figure clearly shows how a spurious divergence can arise in $m_{\mathrm{OSA}}^{*}$ : for instance, within the RPA the denominator in Eq. (38) has a zero at $r_{s} \simeq 15.5$ (see the inset in Fig. 8). Our numerical evidence, within the three theories we have studied, is that, indeed, (i) $\partial_{\omega} \operatorname{Re} \Sigma_{\text {ret }}^{\mathrm{R}}\left(k_{F}, 0\right)$ is negative as it should be for a normal Fermi liquid and monotonically increasing in absolute value as a function of $r_{s}$ and (ii) $\partial_{k} \operatorname{Re} \Sigma_{\text {ret }}^{\mathrm{R}}\left(k_{F}, 0\right)$ is positive and monotonically increasing too. Within the theory outlined in this work, which uses as a key ingredient the Kukkonen-Overhauser effective screening function in Eq. (4), the effect of a charge-only local field is to shift this divergence to higher values of $r_{s}$, while the opposite occurs when including both charge and spin fluctuations. For instance, within the $G_{+} \& G_{-} /$OSA theory the divergence occurs near $r_{s}=5$. Within the local approximation of Eq. (16) the situation is different ${ }^{69}$ and the effect of a charge-only local field is to shift the divergence to lower values of $r_{s}$.

In Fig. 9 we show our numerical results for the renormalization constant $Z$ in comparison to the QMC data of Ref. 70. The theory underestimates the value of $Z$ over the whole range of densities explored. Note that short-range chargedensity fluctuations tend to stabilize the normal Fermi liquid, 

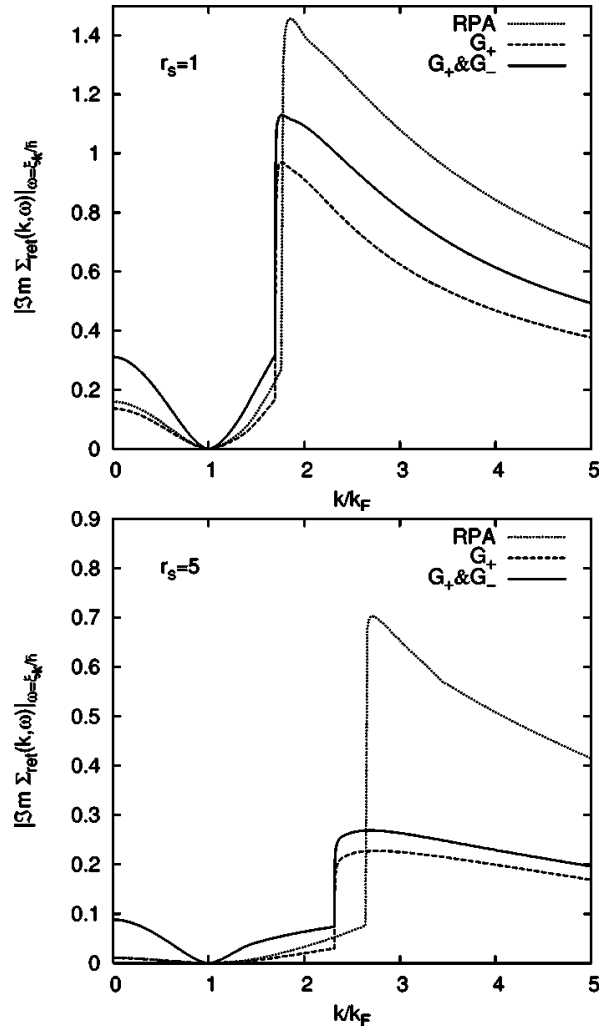

FIG. 6. The absolute value of the imaginary part of the retarded self-energy (in units of Ry) evaluated at $\omega=\xi_{\mathbf{k}} / \hbar$ as a function of $k / k_{F}$ for $r_{s}=1$ (top panel) and 5 (bottom panel).

while the simultaneous inclusion of charge- and spin-density fluctuations works in the opposite way.

For the sake of completeness we have collected in Table I a summary of our numerical results for $m^{*}$ and $Z$ at a few values of $r_{s}$. In Sec. V we will discuss our results for the QP effective mass in the light of recent experimental results and draw our main conclusions.

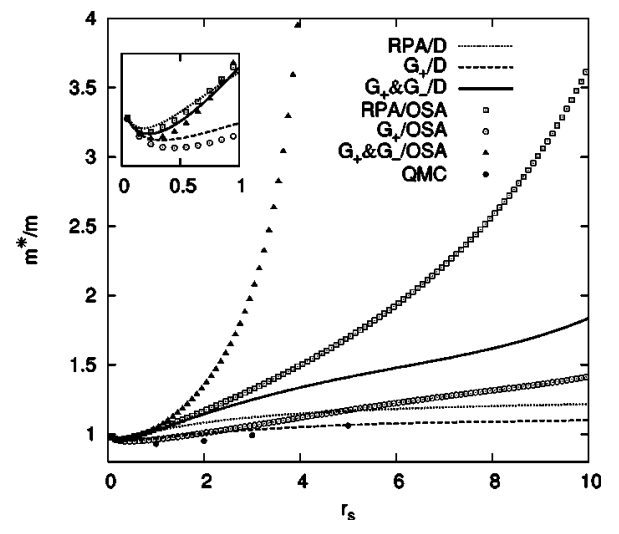

FIG. 7. Effective mass enhancement as a function of $r_{s}$ for 0 $\leqslant r_{s} \leqslant 10$. The inset shows an enlargement of the results for $r_{s} \leqslant 1$. The lines show the results from Eq. (36), while the symbols (except for the dots) are from Eq. (38). The QMC data (dots) are from Ref. 11.

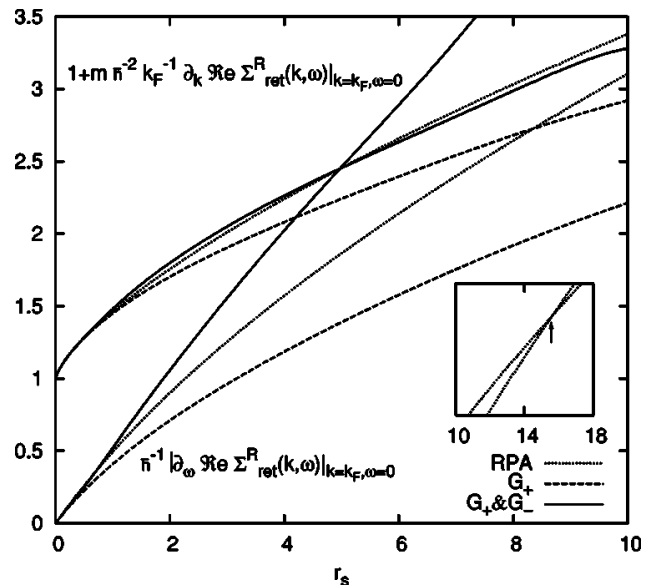

FIG. 8. Illustrating the divergence of the effective mass within the OSA. The three curves starting from unity at $r_{s}=0$ refer to the quantity $1+\left(m / \hbar^{2} k_{F}\right) \partial_{k} \operatorname{Re} \Sigma_{\text {ret }}^{\mathrm{R}}\left(k_{F}, 0\right)$, and the other three curves to $\hbar^{-1}\left|\partial_{\omega} \operatorname{Re} \Sigma_{\text {ret }}^{\mathrm{R}}\left(k_{F}, 0\right)\right|$. The intersection of two lines with the same line style in the two sets of curves corresponds to a zero in the denominator of Eq. (38) and thus to a divergence in $m_{\text {OSA }}^{*}$. The inset shows this divergence occurring within the RPA at $r_{s} \simeq 15.5$.

\section{COMPARISON TO EXPERIMENTAL RESULTS AND CONCLUSIONS}

A full analysis of the published data for the effective mass of carriers in Si-MOSFET's ${ }^{47,48}$ would require a more complete theoretical study, mainly to account for the two-valley nature of the material. We will focus here instead on the experimental results of Ref. 56 as kindly provided to us by Dr. Zhu prior to publication. At present the data refer to the range $2 \lesssim r_{s} \lesssim 6$, so that we cannot judge the performance of the theory in the weak-coupling regime. A quantitative comparison between theory and experiment would also require a refined treatment of a series of effects, such as those due to disorder and to finite temperature. We restrict our analysis to the effect of finite sample thickness, by discussing how a softened Coulomb potential modifies $m^{*}$ against the strictly $2 \mathrm{D}$ results discussed in Sec. IV B and shown in Fig. 7. The expectation is that the QP effective mass will be noticeably smaller when a softened Coulomb interaction is at work.

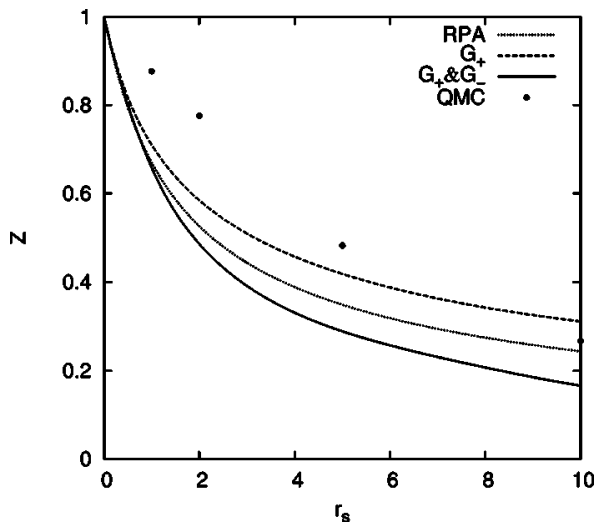

FIG. 9. Renormalization constant $Z$ as a function of $r_{s}$ for 0 $\leqslant r_{s} \leqslant 10$. The QMC data have been taken from Ref. 70 . 
TABLE I. QP effective mass and renormalization constant of a paramagnetic 2D EG. The arrows refer to the divergence in the OSA as explained in the main text.

\begin{tabular}{ccccc}
\hline \hline$r_{s} \quad$ Various calculations & $m_{\mathrm{D}}^{*} / m$ & $m_{\mathrm{OSA}}^{*} / m$ & \multicolumn{2}{l}{$Z$} \\
\hline 1 & $\mathrm{RPA}$ & 1.022 & 1.033 & 0.670 \\
& $G_{+}$ & 0.972 & 0.961 & 0.710 \\
& $G_{+} \& G_{-}$ & 1.026 & 1.040 & 0.658 \\
& $\mathrm{RPA}$ & 1.082 & 1.168 & 0.526 \\
& $G_{+}$ & 1.004 & 1.007 & 0.585 \\
& $G_{+} \& G_{-}$ & 1.144 & 1.349 & 0.486 \\
& $\mathrm{RPA}$ & 1.121 & 1.322 & 0.444 \\
& $G_{+}$ & 1.030 & 1.061 & 0.510 \\
& $G_{+} \& G_{-}$ & 1.247 & 2.026 & 0.391 \\
5 & $\mathrm{RPA}$ & 1.167 & 1.696 & 0.349 \\
& $G_{+}$ & 1.066 & 1.172 & 0.419 \\
& $G_{+} \& G_{-}$ & 1.410 & $\nearrow$ & 0.289 \\
& $\mathrm{RPA}$ & 1.215 & 3.650 & 0.244 \\
& $G_{+}$ & 1.100 & 1.415 & 0.311 \\
& $G_{+} \& G_{-}$ & 1.834 & $\nearrow$ & 0.166 \\
\hline \hline
\end{tabular}

We have thus recalculated $m^{*}$ after renormalizing the bare Coulomb potential by means of a form factor to take into account the finite width of the EG in the GaAs/AlGaAs hetero-junction-insulated gate field-effect transistor used in Refs. 51 and 56. The appropriate renormalized potential is given by $V_{\mathbf{q}}=v_{\mathbf{q}} F(q d) / \bar{\kappa}$, where

$$
F(x)=\left(1+\frac{\kappa_{\mathrm{ins}}}{\kappa_{\mathrm{sc}}}\right) \frac{8+9 x+3 x^{2}}{16(1+x)^{3}}+\left(1-\frac{\kappa_{\mathrm{ins}}}{\kappa_{\mathrm{sc}}}\right) \frac{1}{2(1+x)^{6}},
$$

with $d=\left[\hbar^{2} \kappa_{\mathrm{sc}} /\left(48 \pi m e^{2} n^{*}\right)\right]^{1 / 3}$ representing an effective width of the $2 \mathrm{D} \mathrm{EG} .{ }^{37}$ Here $\kappa_{\text {ins }}=10.9$ and $\kappa_{\mathrm{sc}}=12.9$ are the dielectric constants of the insulator and of the space charge layer, $\bar{\kappa}$ is their average, $m$ is the bare band mass in the confinement direction, and $n^{*}=n_{\mathrm{depl}}+11 n_{2 \mathrm{D}} / 32$, the depletion layer charge density $n_{\text {depl }}$ being zero in the experiments of Ref. 56. The results that we obtain with the softened potential are shown in Fig. 10. A caveat to keep in mind is that we have used the same local-field factors as a zero-thickness 2D EG in the lack of a better choice. Thus the results labeled by $G_{+}$and $G_{+} \& G_{-}$in Fig. 10 contain the effect of finite thickness only through the renormalization of the Coulomb potential. We believe that the explicit dependence of the local fields on the finite width of the 2D EG should not change the results of Fig. 10 in a substantial manner.

Comparing the results of Fig. 10 with those in Ref. 56 we can draw the following conclusions: (i) the $G_{+}$results, at both the OSA and the Dyson-equation level, do not have the proper functional shape to account for the experiment data; (ii) the RPA and $G_{+} \& G_{-}$results are rather similar; and (iii) the $G_{+} \& G_{-}$results, which treat charge and spin fluctuations on the same footing, show the best performance against the experiment data. In fact, without the use of any fitting parameters, the $G_{+} \& G_{-}$results within the OSA compare in a

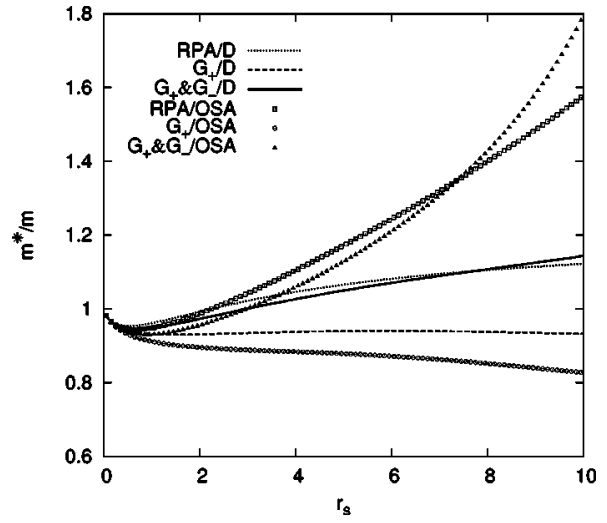

FIG. 10. Effective mass enhancement for a quasi-2D EG confined in a GaAs/AlGaAs triangular quantum well of the type used in Refs. 51 and 56. The notation is as in Fig. 7.

very reasonable manner with the data. The Dyson-equation results, show instead a relatively small and slowly increasing mass enhancement over the whole range of densities, as discussed for the strictly 2D case in Sec. IV B.

In summary, we have revisited the problem of the microscopic calculation of the quasiparticle self-energy and manybody effective mass enhancement in a 2D EG. We have performed a systematic study based on the many-body localfields theory, taking advantage of the results of the most recent diffusion Monte Carlo calculations of the static charge and spin response of the EG expressed through static localfield factors. We have carried out extensive calculations of both the real and imaginary parts of the quasiparticle selfenergy. We have also presented results for the effective mass enhancement and for the renormalization constant over a wide range of coupling strength. In this respect we have critically examined the merits of the on-shell approximation versus the Dyson-equation calculation. Depending on the local-field factors, the OSA predicts a spurious divergence of the effective mass at strong coupling, and a solution of the Dyson equation is therefore necessary in order to obtain the correct value of the effective mass within Fermi-liquid theory. The comparison to the experiment data of Ref. 56 shows that, as already evident even in the higher density regime $^{24,27,28,32}$ of a MOSFET, the simultaneous inclusion of charge- and spin-density fluctuations beyond the random phase approximation is crucial in accounting for exchange and short-range correlations and can lead to substantial corrections at low carrier densities. A possible role of dynamic correlations, entering through the frequency dependence of the local-field factors, remains to be examined.

\section{ACKNOWLEDGMENTS}

We would like to gratefully acknowledge the early contributions to this project by Ermanno Strepparola. We are indebted to George Simion for pointing out to us the existence of the ultraviolet divergence in the calculation of the selfenergy within the static many-body local-fields theory. We would also like to thank J. Zhu, M. Shayegan, Y.-W. Tan, and K. Vakili for sharing with us their considerable physical in- 


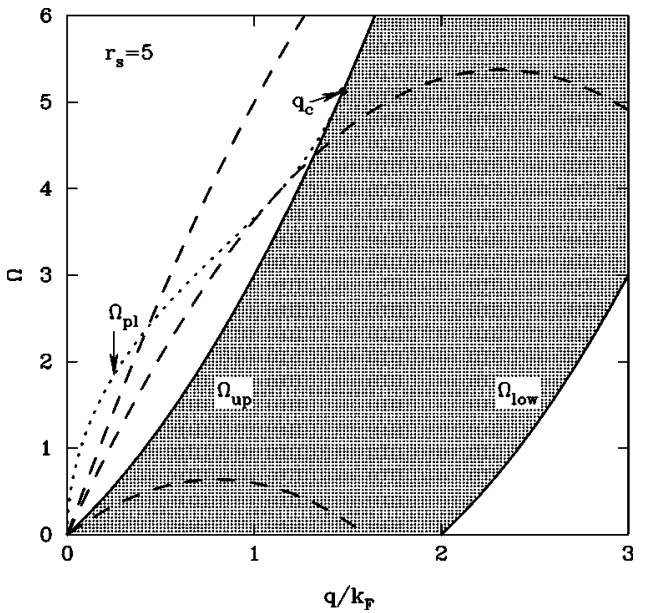

FIG. 11. Illustrating the integration range relevant to Eq. (A4), with $\Omega$ in units of $\varepsilon_{F} / \hbar$. The shaded area represents the electronhole continuum. $\Omega_{\min }(q)$ is shown for three values of $k$ (dashed curves): from top to bottom, $k=3.0 k_{F}, k_{p}$, and $0.8 k_{F}$ with $k_{p}$ $\simeq 2.32 k_{F}$. The plasmon dispersion relation $\Omega_{\mathrm{pl}}(q)$ (dotted curve) is also shown.

sight and their experimental results. Saverio Moroni provided useful clarifications concerning the QMC results. This work was partially supported by MIUR through the PRIN2001 and PRIN2003 programs. G.V. acknowledges support from NSF Grant No. DMR 0313681.

\section{APPENDIX A: DETAILS ON THE EXPLICIT CALCULATION OF THE REAL PART OF THE CH CONTRIBUTION}

Using Eq. (3) we find that the real part of the $\mathrm{CH}$ term evaluated at $\omega=\xi_{\mathbf{k}} / \hbar$ is given by

$$
\begin{aligned}
\left.\operatorname{Re} \Sigma_{\mathrm{CH}}(\mathbf{k}, \omega)\right|_{\omega=\xi_{\mathbf{k}} / \hbar}= & -\int \frac{d^{2} \mathbf{q}}{(2 \pi)^{2}} v_{\mathbf{q}} \mathrm{P} \int_{0}^{+\infty} \frac{d \Omega}{\pi} \\
& \times \frac{\operatorname{Im}\left[\varepsilon^{-1}(\mathbf{q}, \Omega)\right]}{\xi_{\mathbf{k}} / \hbar-\xi_{\mathbf{k}+\mathbf{q}} / \hbar-\Omega} .
\end{aligned}
$$

The angular integration can be performed analytically, with the result

$$
\int_{0}^{2 \pi} \frac{d \theta}{\xi_{\mathbf{k}} / \hbar-\xi_{\mathbf{k}+\mathbf{q}} / \hbar-\Omega}=\frac{2 \pi \Theta\left[\Omega-\Omega_{\min }(k)\right]}{\sqrt{\left[\Omega+\hbar q^{2} /(2 m)\right]^{2}-\hbar^{2} k^{2} q^{2} / m^{2}}}
$$

where $\Omega_{\min }(k)=-\hbar q^{2} /(2 m)+\hbar k q / m$. In carrying out the frequency integration care must be taken to include the contribution from the plasmon pole $\Omega_{\mathrm{pl}}$. Using the expression for the imaginary part of the charge-charge susceptibility near $\Omega_{\mathrm{pl}}$,

$$
\begin{aligned}
\operatorname{Im} \chi_{\mathrm{C}}(\mathbf{q}, \Omega)= & \left.\pi v_{\mathrm{C}}^{-1}(\mathbf{q}) \operatorname{Re} \chi_{0}(\mathbf{q}, \Omega)\right|_{\Omega=\Omega_{\mathrm{pl}}} \\
& \times\left[\left.\frac{\partial \operatorname{Re} \chi_{0}(\mathbf{q}, \Omega)}{\partial \Omega}\right|_{\Omega=\Omega_{\mathrm{pl}}}\right]^{-1} \delta\left(\Omega-\Omega_{\mathrm{pl}}\right),
\end{aligned}
$$

we find that the real part of the $\mathrm{CH}$ term is given by

$$
\begin{aligned}
\left.\operatorname{Re} \Sigma_{\mathrm{CH}}(\mathbf{k}, \omega)\right|_{\omega=\xi_{\mathbf{k}} / \hbar}= & -\int_{0}^{q_{\mathrm{c}}} \frac{q d q}{2 \pi} \frac{\left.v_{\mathrm{C}}(\mathbf{q}) \operatorname{Re} \chi_{0}(\mathbf{q}, \Omega)\right|_{\Omega=\Omega_{\mathrm{pl}}}}{\sqrt{\left[\Omega_{\mathrm{pl}}+\hbar q^{2} /(2 m)\right]^{2}-\hbar^{2} k^{2} q^{2} / m^{2}}}\left[\left.\frac{\partial \operatorname{Re} \chi_{0}(\mathbf{q}, \Omega)}{\partial \Omega}\right|_{\Omega=\Omega_{\mathrm{pl}}}\right]^{-1} \Theta\left[\Omega_{\mathrm{pl}}-\Omega_{\mathrm{min}}(k)\right] \\
& -\int_{0}^{+\infty} \frac{q d q}{2 \pi} \mathrm{P} \int_{\max \left[0, \Omega_{\min }(k), \Omega_{\mathrm{low}}\right]}^{\Omega_{\mathrm{up}}} \frac{d \Omega}{\pi} \frac{v_{\mathrm{C}}^{2}(\mathbf{q}) \operatorname{Im} \chi_{\mathrm{C}}(\mathbf{q}, \Omega)+3 v_{\mathrm{S}}^{2}(\mathbf{q}) \operatorname{Im} \chi_{\mathrm{S}}(\mathbf{q}, \Omega)}{\sqrt{\left[\Omega+\hbar q^{2} /(2 m)\right]^{2}-\hbar^{2} k^{2} q^{2} / m^{2}}} .
\end{aligned}
$$

Here $q_{\mathrm{c}}$ marks the onset of Landau damping and $\Omega_{\text {up(low) }}$ $=\hbar q^{2} /(2 m) \pm \hbar q k_{F} / m$ are the upper and lower edges of the electron-hole continuum.

The range of the momentum integration deserves special attention. In the first term in Eq. (A4), due to the step function the range of $q$-integration is determined by the intersections between $\Omega_{\min }(k)$ and $\Omega_{\mathrm{pl}}$ (see Fig. 11). In Sec. IV A we have introduced the $r_{s}$-dependent wave number $k_{p}$; this is the wave number $k$ at which $\Omega_{\min }(k)$ is tangent to $\Omega_{\mathrm{pl}}$. There are two cases: (i) for $k<k_{p}$ there are no intersections and, thus, the range of $q$ integration goes from 0 to $q_{c}$; and (ii) for $k$ $\geqslant k_{p}$ there can be either one $\left(q_{1}\right)$ or two intersections $\left(q_{1,2}\right)$, so that the range of integration is $\left[0, q_{1}\right]$ or $\left[0, q_{1}\right] \cup\left[q_{2}, q_{c}\right]$, respectively. It is the crossover from condition (i) to (ii) that leads to the plasmon dip in $\left.\operatorname{Re} \Sigma_{\mathrm{CH}}(k, \omega)\right|_{\omega=\xi_{\mathbf{k}} / \hbar}$.

In the second term in Eq. (A4) the range of $q$ integration runs up to $q=+\infty$ and this gives rise to the logarithmic divergence discussed in Ref. 36 and briefly mentioned in Ref. 64. As already shown in Sec. II, what matters are self-energy differences, which are free of singularities. Numerically, we deal only with the finite quantity $\left.\operatorname{Re} \Sigma_{\mathrm{CH}}(\mathbf{k}, \omega)\right|_{\omega=\xi_{\mathbf{k}} / \hbar}$ $-\Sigma_{\mathrm{CH}}\left(k_{F}, 0\right)$. 


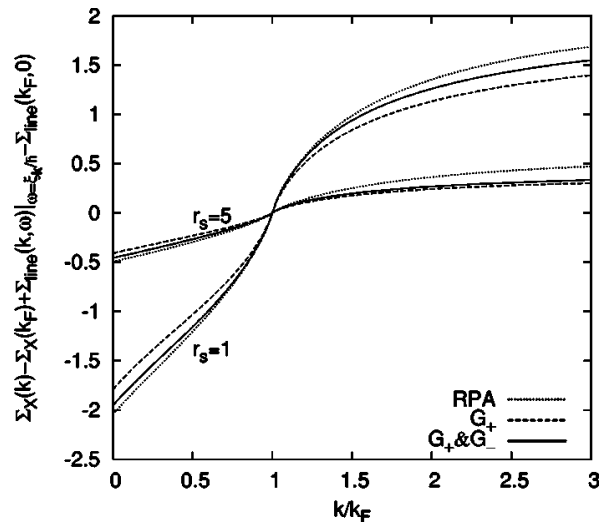

FIG. 12. The exchange plus regularized "line" contribution to the retarded self-energy (in units of Ryd) evaluated at $\omega=\xi_{\mathbf{k}} / \hbar$, as a function of $k / k_{F}$ for $r_{s}=1$ and 5 .

\section{APPENDIX B: LINE+RESIDUE DECOMPOSITION}

In this appendix we discuss a mathematically equivalent decomposition of the QP self-energy, introduced by Quinn and Ferrell, ${ }^{20}$ which has been often employed in the literature. ${ }^{3}$ This amounts to writing

$$
\Sigma_{\text {ret }}(\mathbf{k}, \omega)=\Sigma_{X}(\mathbf{k})+\Sigma_{\text {line }}(\mathbf{k}, \omega)+\Sigma_{\text {res }}(\mathbf{k}, \omega) .
$$

Here the first term is the Hartree-Fock self-energy ${ }^{58}$
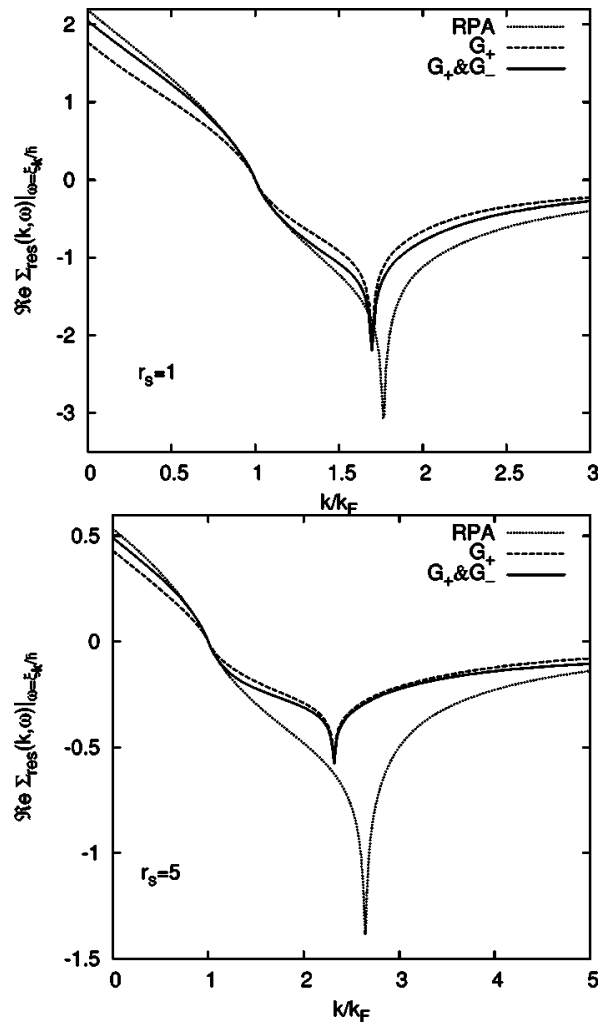

FIG. 13. The real part of the "residue" contribution to the retarded self-energy (in units of Ry) evaluated at $\omega=\xi_{\mathbf{k}} / \hbar$ as a function of $k / k_{F}$ for $r_{s}=1$ (top panel) and 5 (bottom panel).

$$
\Sigma_{\mathrm{X}}(\mathbf{k})=\left\{\begin{array}{ll}
-2 e^{2} k_{F} \mathrm{E}\left(\bar{k}^{2}\right) / \pi & (\bar{k} \leqslant 1) \\
-2 e^{2} k_{F} \bar{k}\left[\mathrm{E}\left(1 / \bar{k}^{2}\right)-\left(1-1 / \bar{k}^{2}\right) \mathrm{K}\left(1 / \bar{k}^{2}\right)\right] / \pi & (\bar{k}>1)
\end{array},\right.
$$

where $\bar{k}=k / k_{F}$ and $\mathrm{K}(x), \mathrm{E}(x)$ are complete elliptical integrals of the first and second kind, respectively. The second term in Eq. (B1), which is purely real, is given by

$$
\begin{aligned}
\Sigma_{\text {line }}(\mathbf{k}, \omega)= & -\int \frac{d^{2} \mathbf{q}}{(2 \pi)^{2}} v_{\mathbf{q}} \int_{-\infty}^{\infty} \frac{d \Omega}{2 \pi}\left[\frac{1}{\varepsilon(\mathbf{q}, i \Omega)}-1\right] \\
& \times \frac{1}{\omega+i \Omega-\xi_{\mathbf{k}+\mathbf{q}} / \hbar} .
\end{aligned}
$$

Finally, the third term is the so-called residue contribution,

$$
\begin{aligned}
\Sigma_{\text {res }}(\mathbf{k}, \omega)= & \int \frac{d^{2} \mathbf{q}}{(2 \pi)^{2}} v_{\mathbf{q}}\left[\frac{1}{\varepsilon\left(\mathbf{q}, \omega-\xi_{\mathbf{k}+\mathbf{q}} / \hbar\right)}-1\right] \\
& \times\left[\Theta\left(\omega-\xi_{\mathbf{k}+\mathbf{q}} / \hbar\right)-\Theta\left(-\xi_{\mathbf{k}+\mathbf{q}} / \hbar\right)\right] .
\end{aligned}
$$

Within this decomposition it is the "line" contribution that needs to be regularized for an ultraviolet divergence.

As a check of our numerical results obtained by means of the SX-CH decompositions, we have recalculated the QP self-energy, effective mass, and renormalization constant by this alternative route. This turned out to require a substantially harder numerical effort. For completeness we summarize in Figs. 12 and 13 our results for the line and residue terms. 
*Present address: Département de Physique and Centre de Recherche en Physique du Solide, Université de Sherbrooke, Sherbrooke, Québec, Canada J1K 2R1.

†E-mail address: m.polini@sns.it

${ }^{1}$ D. M. Ceperley, Nature (London) 397, 386 (1999).

${ }^{2}$ D. Pines and P. Noziéres, The Theory of Quantum Liquids (Benjamin, New York, 1966).

${ }^{3}$ G. F. Giuliani and G. Vignale, Quantum Theory of the Electron Liquid (Cambridge University Press, Cambridge, England, 2005).

${ }^{4}$ K. S. Singwi and M. P. Tosi, in Solid State Physics, edited by H. Ehrenreich, F. Seitz, and D. Turnbull (Academic, New York, 1981), Vol. 36, p. 177.

${ }^{5}$ A. W. Overhauser, Phys. Rev. B 3, 1888 (1971).

${ }^{6}$ N. H. March, Electron Correlation in the Solid State (Imperial College Press, London, 1999).

${ }^{7}$ E. P. Wigner, Phys. Rev. 46, 1002 (1934).

${ }^{8}$ D. M. Ceperley and B. J. Alder, Phys. Rev. Lett. 45, 566 (1980); B. J. Alder, D. M. Ceperley, and E. L. Pollock, Int. J. Quantum Chem. 16, 49 (1982).

${ }^{9}$ B. Tanatar and D. M. Ceperley, Phys. Rev. B 39, 5005 (1989).

${ }^{10}$ S. Moroni, D. M. Ceperley, and G. Senatore, Phys. Rev. Lett. 69, 1837 (1992).

11 Y. Kwon, D. M. Ceperley, and R. M. Martin, Phys. Rev. B 50, 1684 (1994).

${ }^{12}$ S. Moroni, D. M. Ceperley, and G. Senatore, Phys. Rev. Lett. 75, 689 (1995).

${ }^{13}$ F. Rapisarda and G. Senatore, Aust. J. Phys. 49, 161 (1996).

${ }^{14}$ G. Ortiz, M. Harris, and P. Ballone, Phys. Rev. Lett. 82, 5317 (1999).

${ }^{15}$ G. Senatore, S. Moroni, and D. M. Ceperley, in Quantum Monte Carlo Methods in Physics and Chemistry, edited by M. P. Nightingale and C. J. Umrigar (Kluwer, Dordrecht, 1999).

${ }^{16}$ D. Varsano, S. Moroni, and G. Senatore, Europhys. Lett. 53, 348 (2001).

${ }^{17}$ F. H. Zong, C. Lin, and D. M. Ceperley, Phys. Rev. E 66, 036703 (2002).

${ }^{18}$ C. Attaccalite, S. Moroni, P. Gori-Giorgi, and G. B. Bachelet, Phys. Rev. Lett. 88, 256601 (2002).

${ }^{19}$ L. D. Landau, Sov. Phys. JETP 3, 920 (1957).

${ }^{20}$ J. J. Quinn and R. A. Ferrell, Phys. Rev. 112, 812 (1958).

${ }^{21}$ T. M. Rice, Ann. Phys. (N.Y.) 31, 100 (1965).

${ }^{22}$ J. Hubbard, Proc. R. Soc. London, Ser. A 240, 739 (1957) and 243, 336 (1957).

${ }^{23}$ C. S. Ting, T. K. Lee, and J. J. Quinn, Phys. Rev. Lett. 34, 870 (1975); see also T. K. Lee, C. S. Ting, and J. J. Quinn, Solid State Commun. 16, 1309 (1975).

${ }^{24}$ S. Yarlagadda and G. F. Giuliani, Phys. Rev. B 49, 14188 (1994).

${ }^{25}$ G. Vignale and K. S. Singwi, Phys. Rev. B 32, 2156 (1985).

${ }^{26}$ X. Zhu and A. W. Overhauser, Phys. Rev. B 33, 925 (1986).

${ }^{27}$ S. Yarlagadda and G. F. Giuliani, Solid State Commun. 69, 677 (1989).

${ }^{28}$ S. Yarlagadda and G. F. Giuliani, Phys. Rev. B 49, 7887 (1994) and 61, 12556 (2000).

${ }^{29}$ C. A. Kukkonen and A. W. Overhauser, Phys. Rev. B 20, 550 (1979).

${ }^{30}$ D. R. Hamann and A. W. Overhauser, Phys. Rev. 143, 183 (1966)

${ }^{31}$ S. Rahman and G. Vignale, Phys. Rev. B 30, 6951 (1984).

${ }^{32}$ G. E. Santoro and G. F. Giuliani, Phys. Rev. B 39, 12818 (1989).
${ }^{33}$ S. Yarlagadda and G. F. Giuliani, Phys. Rev. B 40, 5432 (1989).

${ }^{34}$ T. K. Ng and K. S. Singwi, Phys. Rev. B 34, 7738 (1986); 34, 7743 (1986).

${ }^{35}$ B. Davoudi, M. Polini, G. F. Giuliani, and M. P. Tosi, Phys. Rev. B 64, 153101 (2001); 64, 233110 (2001).

${ }^{36} \mathrm{G}$. Simion and G. F. Giuliani (unpublished).

${ }^{37}$ T. Ando, A. B. Fowler, and F. Stern, Rev. Mod. Phys. 54, 437 (1982).

${ }^{38}$ A. A. Abrikosov, Fundamentals of the Theory of Metals (North Holland, Amsterdam, 1988); E. M. Lifshitz and L. P. Pitaevskii, Physical Kinetics (Nauka, Moscow, 1979).

${ }^{39}$ J. L. Smith and P. J. Stiles, Phys. Rev. Lett. 29, 102 (1972).

${ }^{40}$ G. Abstreiter, J. P. Koothaus, J. F. Koch, and G. Dorda, Phys. Rev. B 14, 2480 (1976).

${ }^{41}$ F. F. Fang and P. J. Stiles, Phys. Rev. 174, 823 (1968).

${ }^{42}$ T. Neugebauer, K. von Klitzing, G. Landwehr, and G. Dorda, Solid State Commun. 17, 295 (1975).

${ }^{43}$ For a review on the 2D MIT see, e.g., E. Abrahams, S. V. Kravchenko, and M. P. Sarachik, Rev. Mod. Phys. 73, 251 (2001); also see B. L. Altshuler, D. L. Maslov, and V. M. Pudalov, Physica E (Amsterdam) 9, 209 (2001).

${ }^{44}$ V. T. Dolgopolov, G. V. Kravchenko, A. A. Shashkin, and S. V. Kravchenko, JETP Lett. 55, 733 (1992); D. Simonian, S. V. Kravchenko, M. P. Sarachik, and V. M. Pudalov, Phys. Rev. Lett. 79, 2304 (1997); V. M. Pudalov, G. Brunthaler, A. Prinz, and G. Bauer, JETP Lett. 65, 932 (1997).

${ }^{45}$ T. Okamoto, K. Hosoya, S. Kawaji, and A. Yagi, Phys. Rev. Lett. 82, 3875 (1999).

${ }^{46}$ S. A. Vitkalov, H. Zheng, K. M. Mertes, M. P. Sarachik, and T. M. Klapwijk, Phys. Rev. Lett. 87, 086401 (2001).

${ }^{47}$ A. A. Shashkin, S. V. Kravchenko, V. T. Dolgopolov, and T. M. Klapwijk, Phys. Rev. Lett. 87, 086801 (2001) and Phys. Rev. B 66, 073303 (2002).

${ }^{48}$ V. M. Pudalov, M. E. Gershenson, H. Kojima, N. Butch, E. M. Dizhur, G. Brunthaler, A. Prinz, and G. Bauer, Phys. Rev. Lett. 88, 196404 (2002); see also V. M. Pudalov, M. E. Gershenson and H. Kojima, cond-mat/0110160.

${ }^{49}$ E. Tutuc, S. Melinte, and M. Shayegan, Phys. Rev. Lett. 88, 036805 (2002).

${ }^{50}$ H. Noh, M. P. Lilly, D. C. Tsui, J. A. Simmons, E. H. Hwang, S. Das Sarma, L. N. Pfeiffer, and K. W. West, Phys. Rev. B 68, 165308 (2003).

${ }^{51}$ J. Zhu, H. L. Stormer, L. N. Pfeiffer, K. W. Baldwin, and K. W. West, Phys. Rev. Lett. 90, 056805 (2003).

${ }^{52}$ K. Vakili, Y. P. Shkolnikov, E. Tutuc, E. P. De Poortere, and M. Shayegan, Phys. Rev. Lett. 92, 226401 (2003).

${ }^{53}$ I. M. Lifshitz and A. M. Kosevich, Sov. Phys. JETP 2, 636 (1956); J. M. Luttinger, Proc. R. Soc. Med. 121, 1251 (1961); Yu. A. Bychkov and L. P. Gor'kov, Sov. Phys. JETP 14, 1132 (1962).

${ }^{54}$ K. Miyake and C. M. Varma, Solid State Commun. 85, 335 (1993); S. Curnoe and P. C. E. Stamp, Phys. Rev. Lett. 80, 3312 (1998).

${ }^{55}$ G. W. Martin, D. L. Maslov, and M. Yu. Reizer, Phys. Rev. B 68, 241309(R) (2003).

${ }^{56}$ Y.-W. Tan, J. Zhu, H. L. Stormer, L. N. Pfeiffer, K. W. Baldwin, and K. W. West (private communication).

${ }^{57}$ F. Stern, Phys. Rev. Lett. 18, 546 (1967).

${ }^{58}$ A. V. Chaplik, Sov. Phys. JETP 33, 997 (1971); see also F. Stern, Phys. Rev. Lett. 30, 278 (1973). 
${ }^{59}$ P. Noziéres, Theory of Interacting Fermi Systems (Benjamin, New York, 1964).

${ }^{60}$ L. Hedin, Phys. Rev. 139, A796 (1965).

${ }^{61}$ G. D. Mahan and B. E. Sernelius, Phys. Rev. Lett. 62, 2718 (1989).

${ }^{62}$ H. M. Böhm, S. Conti, and M. P. Tosi, J. Phys.: Condens. Matter 8, 781 (1996); S. Conti, R. Nifosì, and M. P. Tosi, ibid. 9, L475 (1997); R. Nifosì, S. Conti, and M. P. Tosi, Phys. Rev. B 58, 12758 (1998); G. Vignale and W. Kohn, in Electronic Density Functional Theory, Recent Progress and New Directions, edited by J. Dobson, G. Vignale, and M. P. Das (Plenum, New York, 1998), p. 199; Z. Qian and G. Vignale, Phys. Rev. B 65, 235121 (2002); 68, 195113 (2003).

${ }^{63}$ A. Holas, in Strongly Coupled Plasma Physics, edited by F. J. Rogers and H. E. DeWitt (Plenum, New York, 1986), p. 463; G. E. Santoro and G. F. Giuliani, Phys. Rev. B 37, 4813 (1988); G. Vignale, ibid. 38, 6445 (1988).

${ }^{64}$ As it can be demonstrated through a simple power-counting argument, the leading term in the large- $q$ behavior of the static local-field factors in Eq. (32) induces a logarithmic ultraviolet divergence in the real part of $\Sigma_{\mathrm{CH}}$ as given by Eq. (3). Such a divergence is not surprising, for the theory that we are using applies only within a shell of momenta close to the Fermi mo- mentum (see Sec. II C). As discussed in Ref. 36 this formal problem can be handled via an appropriate renormalization procedure. The problem does not affect the QP excitation energy because this quantity depends only on self-energy differences. Obviously, to determine the chemical potential one has to make recourse to a different approach: for instance, $\mu$ can be obtained from parametrizations of the QMC ground-state energy ${ }^{13,18}$ or calculated by refined theories of the ground-state energy [see, e.g., R. Asgari, B. Davoudi, and M. P. Tosi, Solid State Commun. 131, 301 (2004) for the 2D EG].

${ }^{65}$ Y. Isawa and H. Yasuhara, Solid State Commun. 46, 807 (1983); S. Nagano, K. S. Singwi, and S. Ohnishi, Phys. Rev. B 29, 1209 (1984); 31, 3166 (1985); R. Asgari, M. Polini, B. Davoudi, and M. P. Tosi, Solid State Commun. 125, 139 (2003).

${ }^{66}$ G. F. Giuliani and J. J. Quinn, Phys. Rev. B 26, 4421 (1982).

${ }^{67}$ In this case the normal Fermi-liquid assumption breaks down and a singular behavior of $m_{\mathrm{D}}^{*}$ could be interpreted as a quantumphase transition of the 2D EG to a non-Fermi-liquid state.

${ }^{68}$ Y. Zhang and S. Das Sarma, Phys. Rev. B 71, 045322 (2005).

${ }^{69}$ R. Asgari, B. Davoudi, and B. Tanatar, Solid State Commun. 130, 13 (2004).

${ }^{70}$ S. Conti, Ph.D. Thesis, Scuola Normale Superiore, 1997 (unpublished). 